\title{
Polypores of western Finnish Lapland and seasonal dynamics of polypore beetles
}

\author{
DMITRY S. SCHIGEL, TUOMO NIEMELÄ and JUHA KINNUNEN
}

\begin{abstract}
SCHIGEL, D. S., NIEMELÄ, T. \& KINNUNEN, J. 2006: Polypores of western Finnish Lapland and seasonal dynamics of polypore beetles. - Karstenia 46: 37-64. Helsinki. ISSN 0453-3402.

The paper summarizes field inventories of polypores and associated beetles in northwestern Finland (Lapland) in the years 2001-2004. The research area includes nearvirgin or old-growth forests of the Pallas-Yllästunturi National Park and the Pisavaara Strict Nature Reserve, one of the largest areas of protected forests in the country. Altogether 132 polypore species were found or (a few) documented from herbarium collections. Special attention was paid to those beetles that visit or inhabit polypores, either to consume their spores and mycelium, or living in polypore basidiocarps at larval stages. Beetle species compositions and their occurrences as larvae versus imagines differed according to the characteristics of polypore basidiomata. Four kinds (life cycles) of basidiomata were separated: ephemeral, annual sturdy, annual hibernating, and perennial. The selective effect of these life cycles over attracted beetles is extensively discussed with numerous examples. Parallels were found between the insects living in decayed wood versus mushrooms: while beetles of the ephemeral polypore hosts follow in their biology the "mushroom" pathway (pupating in soil debris), those living in perennial basidiocarps follow the "wood" pathway in both consuming the fungus and in pupating inside the decaying basidiocarp. The paper includes complete lists of polypores and polypore-linked beetles found in the study area. A number of wood-inhabiting fungi of other groups are listed, including observations of their beetle fauna. Also more theoretical aspects of the fungus-beetle interaction are discussed.
\end{abstract}

Key words: Basidiomycota, ecology, Coleoptera, Finland, Lapland, basidiocarp, saproxylic, sporulation, fungivory

Dmitry S. Schigel, Tuomo Niemelä, and Juha Kinnunen, Botanical Museum, Finnish Museum of Natural History, P. O. Box 7 (Unioninkatu 44), FI-00014 University of Helsinki, Finland. Correspondence e-mail: dmitry.shchigel@helsinki.fi

\section{Introduction}

In 1998 the Finnish Forest and Park Service organized the first large-scale inventory of Finnish poroid basidiomycete fungi (polypores) in protected forests. Started by Niemelä and Dai (1998, 1999) in the Luosto range, the work continued in Korouoma (Niemelä \& Kinnunen 2001) and North Karelian Biosphere Reserve (Niemelä et al. 2002, Schigel et al. 2004), all in northern and eastern Finland. Material collected during recent inventories in the Ylläs-Aakenus area in 2001 and the Pisavaara Strict Nature Reserve in 2003, combined with results from a collecting trip to the Pallas-Ounastunturi National Park in 2004, make the basis for the present paper. Our team has carried out inventories with similar methods also in the Repovesi National Park, Kirjovaara Primeval Forest, and Kolvananuuro Nature Reserve (in 2004), Salla (in 2005) and Kolovesi National Park (in 2006). These sites are shown on Fig. 1.

The Ylläs-Aakenus Natura 2000 Site, now consolidated with the Pallas-Ounastunturi National Park into the Pallas-Yllästunturi National Park, make together with the Pisavaara Strict Na- 


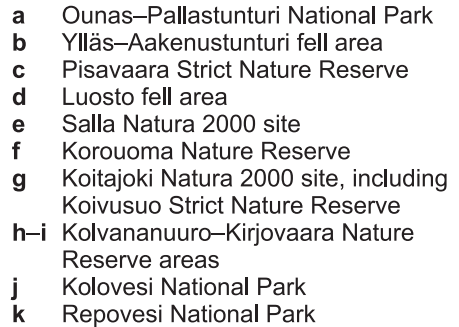

NB Northern Boreal zone (white areas are predominantly fell ranges above the timberline)

MB Middle Boreal zone

SB Southern Boreal zone

HB Hemiboreal zone
Fig. 1. Research areas in Finland $(\mathrm{a}-\mathrm{k})$ inventoried during 1998-2006. Polypore inventory was done with the same method in all these areas. The present paper on polypores deals with areas a-c. Vegetation zones are according to Ahti et al. (1968), zones along the northern coastline not shown.

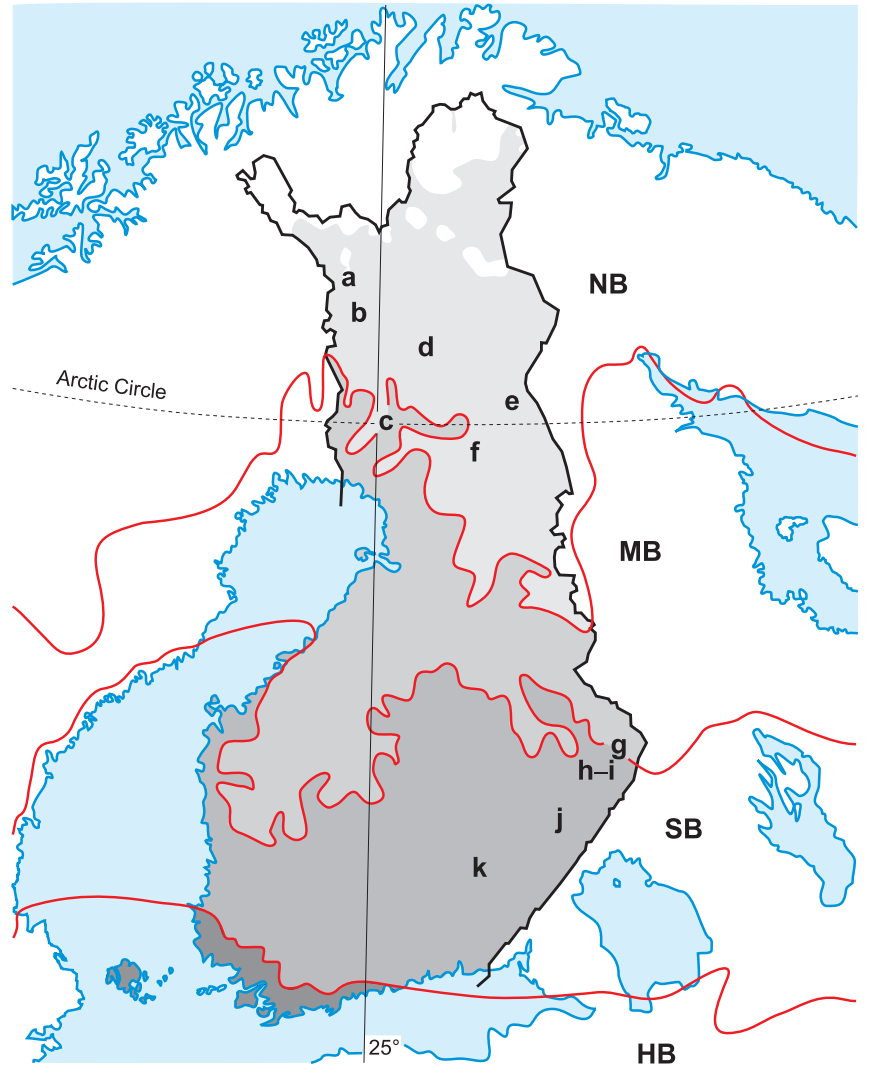

forts put in recent decades in Nordic countries and Russia for better understanding of the ecological relationships between beetles and fungi (also see Schigel et al. 2004, and Nikitsky \& Schigel 2004), the present level of information remains insufficient as to individual fungal species and beetles associated with them.

\section{Materials and methods}

Fungal material from 192 forest compartments (in Finnish metsäkuvio) in the former Ylläs-Aakenus National Park was collected by the authors, Yu-Cheng Dai (Chinese Academy of Sciences) and Olli Turunen (Finnish Nature League). In Pisavaara Strict Nature Reserve in 2003 material was collected by the authors from 124 forest compartments (Niemelä et al. 2005a). In a shorter collecting trip to the former Pallas-Ounastunturi National Park, TN, DSS and Petri Oinonen (University of Helsinki) listed polypores of the studied forests with several hundreds of observations and collections.

An attempt was made to use an up-to-date nomenclature in the scientific names of polypores. In some genera genus and species names have recently been changed, and the reader may meet problems in knowing in which sense (1952), Wegelius (1960), Sippola et al. (1995), and Rintala et al. (2003). In spite of notable ef- 

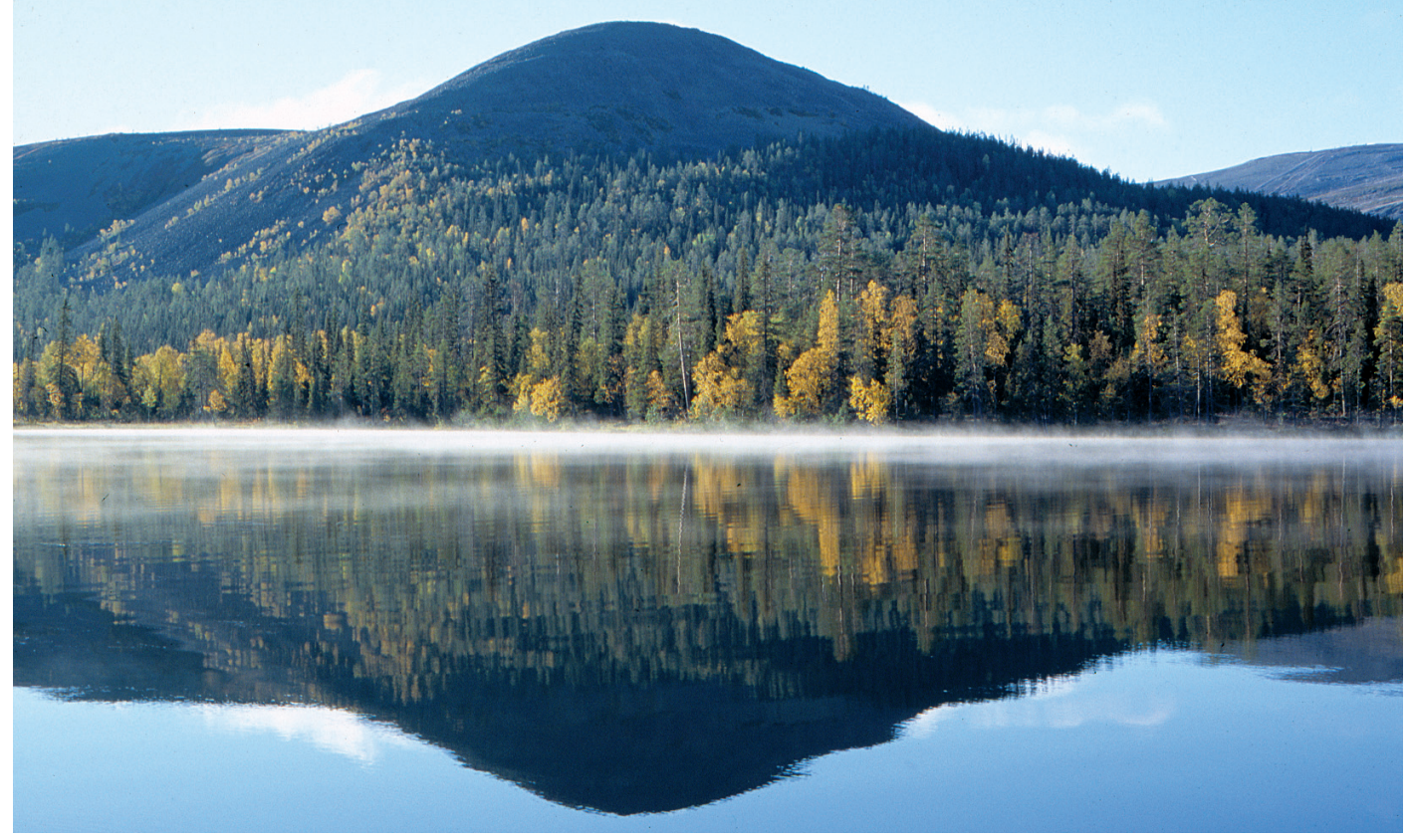

Fig. 2. Frosty morning at lake Kesänkijärvi and Kellostapuli fell in Pallas-Yllästunturi National Park. Photo TN.

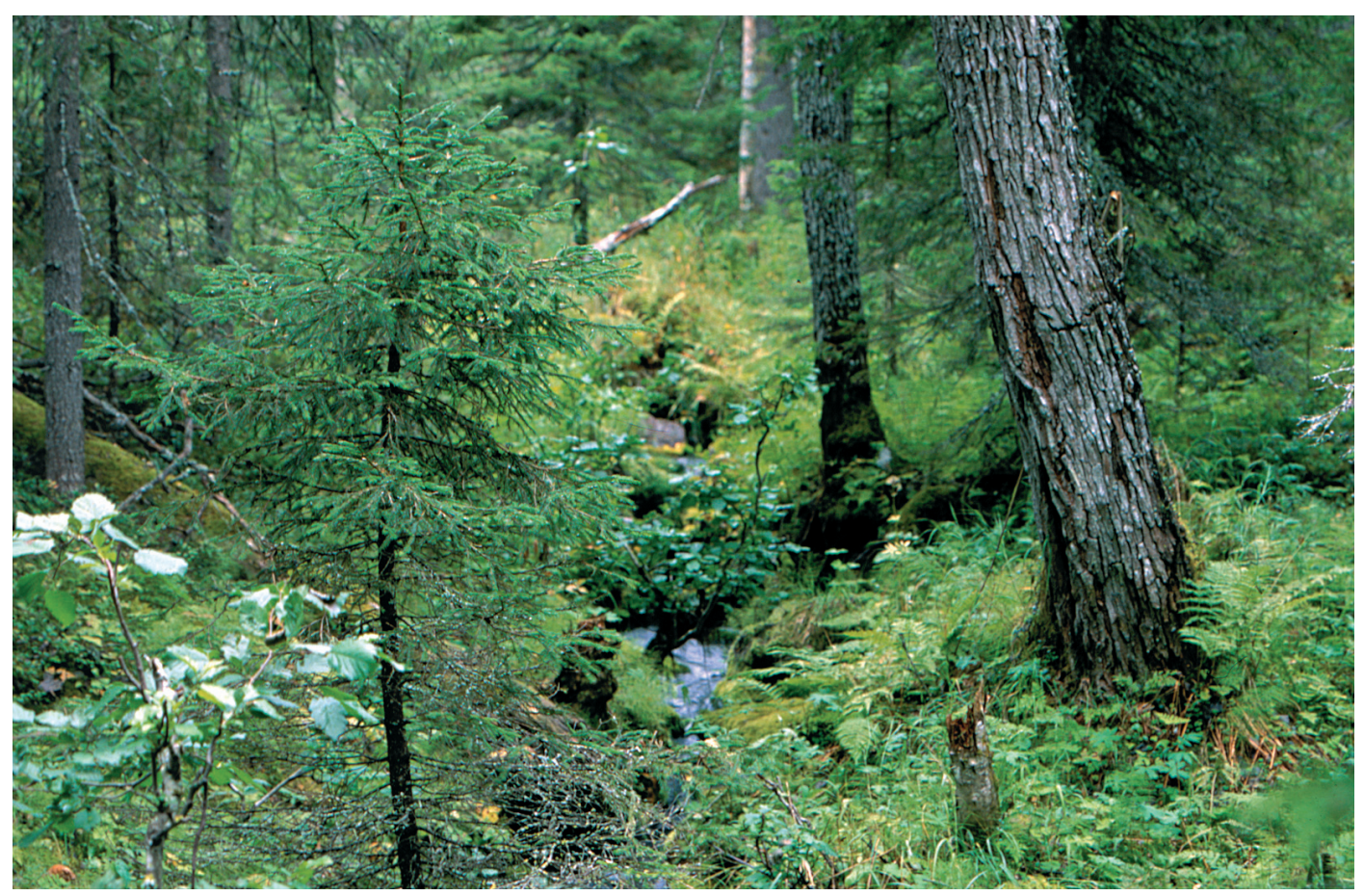

Fig. 3. Forest brook and old-growth spruce forest in a slope. Pisavaara Strict Nature Reserve. Photo DSS. 
they are used in our paper. In Table 1 such species are listed together with their more familiar epithets.

Complete lists of polypores were made for each forest compartment. For a detailed description of this method, see Niemelä et al. (2005a). All insect specimens, plus rare and difficult polypore species were collected for a microscopic investigation. Non-polypore fungi and their insects were also collected when time allowed. Fungal specimens were dried in mushroom dryers with ventilated air at $+40-45^{\circ} \mathrm{C}$. In difficult cases the identifications were later approved by studying sections mounted in Cotton Blue or Melzer's reagent at $\times 1250$ magnification and phase contrast illumination. Fungal collections are preserved in the Herbarium of the Botanical Museum, Finnish Museum of Natural History, University of Helsinki (H); their nomenclature follows Niemelä (2005), except for the Oligoporus-Postia complex, where the species were kept mostly in Postia and only O. sericeomollis and $O$. rennyi in Oligoporus. This genus complex will be treated comprehensively in a forthcoming paper.

Fungal specimens were examined for associated insects, of which larvae and adult beetles were collected separately and preserved in $70 \%$ alcohol for further identification. The fruit bodies of the polypores which contained beetle larvae were collected for rearing. Rearing material collected in summer-autumn was then kept for 2-3 months in outdoor temperature, and exposed to room temperature for further two months before checking the rearing results. Breeding records (Tables 2, 3) meet the criteria set by Lawrence (1973). Coleoptera of the commonest Finnish polypores (Niemelä 2003) were not collected, but in a few cases beetle species of such fungi were noted, and therefore the presented data has a bias against the fauna of common fungi. Beetles will be donated after their investigation to the Zoological Museum, Finnish Museum of Natural History, University of Helsinki; single specimens have been given to identifiers (see Acknowledgements). Beetle nomenclature follows Silfverberg (2004), but the Ciidae are listed according to Müller et al. (2001). Authors of the Latin names of both fungi and beetles are given in Tables 2 and 3 respectively, and they are not repeated in the text.

All authors took part in field work, and DSS collected all insect specimens. This paper was prepared so that DSS wrote most of the text and all entomological sections, JK took part in the proofreading of the manuscript, and TN supervised the writing and wrote sections on fungal taxonomy.

Table 1. Some names of polypores that have been changed recently, and their commoner synonyms or misapplications in contemporary literature.

Name in this paper

Antrodiella canadensis

Antrodiella pallescens

Antrodiella serpula

Ceriporiopsis pseudogilvescens

Cinereomyces lenis

Cinereomyces lindbladii

Fibroporia norrlandica

Ganoderma lipsiense

Hapalopilus aurantiacus

Meruliopsis taxicola

Polyporus leptocephalus

Postia parva (comb. ined.)

Postia hibernica (sensu typi)

Rhodonia placenta

Spongiporus undosus

\section{Commonly used synonyms and misapplications}

Antrodiella overholtsii, Tyromyces canadensis

Antrodiella semisupina auct. (Miettinen et al. 2006)

Antrodiella hoehnelii (Miettinen et al. 2006)

Ceriporiopsis resinascens pro parte (Kinnunen \& Niemelä 2005)

Skeletocutis lenis

Diplomitoporus lindbladii

Oligoporus norrlandicus (Berglund \& Ryvarden 2000, Niemelä et al. 2001)

\section{Ganoderma applanatum}

Hapalopilus salmonicolor auct., Sarcoporia salmonicolor auct. (see Niemelä et al. 2005b)

Gloeoporus taxicola

Polyporus varius

Oligoporus parvus (Renvall 2005), Oligoporus hibernicus auct., Postia hibernica auct.

Oligoporus septentrionalis, Postia septentrionalis (Renvall 2005)

Oligoporus placenta, Postia placenta (Niemelä et al. 2005b)

Oligoporus undosus, Postia undosa (Niemelä et al. 2005b) 
Table 2. Polypores (plus selected other Basidiomycota) and their beetles in western Finnish Lapland. Polypore species are given in an alphabetical order. IUCN = threat categories according to Rassi et al. (2001), indicated in bold face; $\mathrm{L}=$ locality: Y = Ylläs-Aakenus National Park, PV = Pisavaara Strict Nature Reserve, PO = Pallas-Ounastunturi National Park; * = herbarium $(\mathrm{H})$ reference, collected before 2000 (Niemelä \& Kinnunen 2003); localities are also indicated for insect records in bold face; $\mathrm{N}=$ number of compartments where found; $\mathrm{I} / \mathrm{S}=$ number of fungal specimens inhabited by beetles/studied. Numbers of insect specimens found or reared are given in parentheses after the beetle species name. Beetle - fungus associations meeting the Lawrence (1973) criterion are marked with "10+" instead of the number of larvae or reared imago specimens. Adult insects collected in nature indicated in light italics; larvae or reared adults in bold italics. $\dagger=$ literature data on associated beetles by Rintala et al. (2003).

\begin{tabular}{|c|c|c|c|c|c|c|}
\hline & Fungus species & IUCN & $\mathbf{L}$ & $\mathbf{N}$ & $\mathbf{I} / \mathbf{S}$ & Insect records \\
\hline 1 & $\begin{array}{l}\text { Albatrellus confluens (Alb. \& Schwein. : } \\
\text { Fr.) Kotl. \& Pouzar }\end{array}$ & & $\mathrm{Y}, \mathrm{PV}$ & 10 & $0 / 11$ & \\
\hline 2 & $\begin{array}{l}\text { Albatrellus ovinus (Schaeff. : Fr.) Kotl. \& } \\
\text { Pouzar }\end{array}$ & & $\mathrm{Y}, \mathrm{PV}$ & 11 & $0 / 23$ & \\
\hline 3 & Amylocystis lapponica (Romell) Singer & $\mathbf{V U}$ & $\mathrm{PO}, \mathrm{PV}$ & 56 & $8 / 184$ & $\begin{array}{l}\text { PO: Acrulia inflata (1), Sulcacis } \\
\text { fronticornis (1) PV: Hallomenus } \\
\text { binotatus (10+), Sciaridae G. sp. } \\
\text { (3), Sepedophilus littoreus (1), } \\
\text { Quedius plagiatus (1), Ostoma fer- } \\
\text { ruginea (2), Phyllodrepa linearis } \\
\text { (13), Corticaria longicollis (1), } \\
\text { Atomaria affinis (1) PO \& PV: Or- } \\
\text { thoperus rogeri (2) }\end{array}$ \\
\hline 4 & Anomoporia bombycina (Fr.) Pouzar & NT & $\mathrm{Y}, \mathrm{PV}$ & 2 & $0 / 14$ & \\
\hline 5 & $\begin{array}{l}\text { Anomoporia kamtschatica (Parmasto) } \\
\text { M. Bondartseva }\end{array}$ & & $\mathrm{Y}, \mathrm{PV}$ & 4 & $0 / 9$ & \\
\hline 6 & Antrodia albobrunnea (Romell) Ryvarden & NT & $\mathrm{PO}, \mathrm{Y}, \mathrm{PV}$ & 29 & $0 / 93$ & \\
\hline 7 & Antrodia crassa (P. Karst.) Ryvarden & $\mathbf{E N}$ & $\mathrm{Y}, \mathrm{PV}^{*}$ & 2 & $0 / 2$ & \\
\hline 8 & Antrodia infirma Renvall \& Niemelä & $\mathbf{V U}$ & $\mathrm{PO}, \mathrm{Y}, \mathrm{PV}$ & 10 & $6 / 30$ & $\begin{array}{l}\text { PV: Cis micans (1), Phyllodrepa } \\
\text { linearis (4), Rhyncolus ater (4) }\end{array}$ \\
\hline 9 & Antrodia macra (Sommerf.) Niemelä & & PV & 1 & $0 / 1$ & \\
\hline 10 & Antrodia primaeva Renvall \& Niemelä & VU & PV & 2 & $0 / 2$ & \\
\hline 11 & Antrodia pulvinascens (Pilát) Niemela & $\mathbf{V U}$ & $\mathrm{Y}, \mathrm{PV}$ & 6 & $0 / 7$ & \\
\hline 12 & Antrodia serialis (Fr.) Donk & & $\mathrm{PO}, \mathrm{Y}, \mathrm{PV}$ & 68 & $1 / 185$ & $\begin{array}{l}\text { PO: Sulcacis fronticornis (2) } \\
\text { Y: Cis dentatus } \dagger\end{array}$ \\
\hline 13 & Antrodia sinuosa (Fr.) P. Karst. & & $\mathrm{PO}, \mathrm{Y}, \mathrm{PV}$ & 75 & $0 / 138$ & \\
\hline 14 & Antrodia xantha (Fr. : Fr.) Ryvarden & & $\mathrm{PO}, \mathrm{Y}, \mathrm{PV}$ & 69 & $1 / 170$ & PV: Orthoperus rogeri (1) \\
\hline 15 & Antrodiella canadensis (Overh.) Niemelä & $\mathbf{E N}$ & $\mathrm{PV}^{*}$ & 1 & $0 / 1$ & \\
\hline 16 & Antrodiella citrinella Niemelä \& Ryvarden & VU & $\mathrm{Y}, \mathrm{PV}$ & 6 & $0 / 16$ & \\
\hline 17 & Antrodiella faginea Vampola \& Pouzar & & Y, PV & 8 & $0 / 16$ & \\
\hline 18 & $\begin{array}{l}\text { Antrodiella pallasii Renvall, Johannesson } \\
\text { \& Stenlid }\end{array}$ & & $\mathrm{PO}, \mathrm{Y}, \mathrm{PV}$ & 23 & $0 / 72$ & \\
\hline 19 & $\begin{array}{l}\text { Antrodiella pallescens (Pilát) Niemelä \& } \\
\text { Miettinen }\end{array}$ & & $\mathrm{PO}, \mathrm{Y}, \mathrm{PV}$ & 17 & $3 / 40$ & $\begin{array}{l}\text { PV: Cis jacquemartii (2), Sciophila } \\
\text { buxtoni (1 female), Acrulia inflata } \\
\text { (2), Leptusa pulchella (1), Rhizo- } \\
\text { phagus dispar (1) }\end{array}$ \\
\hline 20 & Antrodiella romellii (Donk) Niemelä & & $\mathrm{Y}, \mathrm{PV}$ & 6 & $0 / 13$ & \\
\hline 21 & $\begin{array}{l}\text { Antrodiella serpula (P. Karst.) Spirin \& } \\
\text { Niemelä }\end{array}$ & & $\mathrm{Y}$ & 1 & $0 / 1$ & \\
\hline 22 & Bjerkandera adusta (Willd. : Fr.) P. Karst. & & $\mathrm{PO}, \mathrm{PV}$ & 2 & $0 / 2$ & \\
\hline 23 & Bjerkandera fumosa (Pers. : Fr.) P. Karst. & & PV & 1 & $0 / 1$ & \\
\hline 24 & $\begin{array}{l}\text { Boletopsis grisea (Peck) Bondartsev \& } \\
\text { Singer }\end{array}$ & & Y, PV & 2 & $0 / 2$ & \\
\hline 25 & $\begin{array}{l}\text { Byssoporia mollicula (Bourdot) Larsen \& } \\
\text { Zak }\end{array}$ & & $\mathrm{Y}, \mathrm{PV}$ & 18 & $0 / 31$ & \\
\hline 26 & $\begin{array}{l}\text { Ceriporia reticulata (H. Hoffm. : Fr.) } \\
\text { Domański }\end{array}$ & & $\mathrm{Y}, \mathrm{PV}$ & 6 & $0 / 14$ & \\
\hline 27 & Ceriporia viridans (Berk. \& Broome) Donk & & $\mathrm{Y}, \mathrm{PV}$ & 5 & $0 / 9$ & \\
\hline 28 & $\begin{array}{l}\text { Ceriporiopsis aneirina (Sommerf.) } \\
\text { Domański }\end{array}$ & & PV & 1 & $0 / 3$ & \\
\hline 29 & $\begin{array}{l}\text { Ceriporiopsis pseudogilvescens } \\
\text { (Pilát) Niemelä }\end{array}$ & & $\mathrm{Y}, \mathrm{PV}^{*}$ & 2 & $0 / 7$ & \\
\hline 30 & Cerrena unicolor (Bull. : Fr.) Murrill & & $\mathrm{PO}, \mathrm{Y}, \mathrm{PV}$ & 47 & $0 / 152$ & \\
\hline
\end{tabular}


Cinereomyces lindbladii (Berk.) Jülich

Climacocystis borealis (Fr.) Kotl. \& Pouzar
31 Cinereomyces lenis (P. Karst.) Spirin

VU

$\begin{array}{lll}\text { PO, Y, PV } & 14 & 0 / 58 \\ \text { PO, PV } & 2 & 0 / 10 \\ \text { PO, Y, PV } & 26 & 5 / 64\end{array}$

Coltricia perennis (L. : Fr.) Murrill

35 Daedaleopsis confragosa (Bolton : Fr.)

J. Schröt.

36 Daedaleopsis septentrionalis

(P. Karst.) Niemelä

37 Dichomitus squalens (P. Karst.) D.A. Reid

38 Diplomitoporus crustulinus

(Bres.) Domański

39 Fibroporia norrlandica (Berglund \&

Ryvarden) Niemelä

40 Fomes fomentarius (L. : Fr.) Fr.

41 Fomitopsis pinicola (Sw. : Fr.) P. Karst.

42 Fomitopsis rosea (Alb. \& Schwein. : Fr.)

P. Karst.

43 Ganoderma lipsiense (Batsch) G.F. Atk.

44 Gelatoporia subvermispora (Pilát) Niemelä NT

45 Gloeophyllum odoratum (Wulfen : Fr.)

Imazeki

46 Gloeophyllum protractum (Fr.) Imazeki

47 Gloeophyllum sepiarium (Wulfen: Fr.)

P. Karst.

48 Gloeoporus dichrous (Fr. : Fr.) Bres.

49 Gloeoporus pannocinctus (Romell)

J. Erikss.

50 Hapalopilus aurantiacus (Rostk.) Bondart- NT sev \& Singer

51 Hapalopilus rutilans (Pers. : Fr.) P. Karst.

52 Haploporus odorus (Sommerf.) Bondartsev NT \& Singer

53 Heterobasidion parviporum Niemelä \& Korhonen

$54 \quad$ Inonotopsis subiculosa (Peck) Parmasto

55 Inonotus obliquus (Pers. : Fr.) Pilát

$\begin{array}{llll} & \text { PO, Y, PV } & 21 & 0 / 61 \\ & \text { Y } & 1 & 0 / 1 \\ & \text { PO, Y, PV* } & 5 & 2 / 14 \\ \text { NT } & \text { Y, PV } & 5 & 0 / 12 \\ \text { NT } & \text { Y, PV* } & 4 & 0 / 12 \\ & \text { Y } & 1 & 0 / 1 \\ & & & \\ & \text { PO, Y, PV } & 106 & 2 / 270\end{array}$

NT

PO, Y, PV $111 \quad 1 / 241$

PO, Y, PV $59 \quad 0 / 209$

$\begin{array}{lll}Y, P V & 13 & 0 / 14\end{array}$

$\begin{array}{lll}\mathrm{Y}, \mathrm{PV} & 2 & 0 / 3\end{array}$

$\mathrm{Y}, \mathrm{PV}^{*} \quad 4 \quad 0 / 9$

NT

$\mathrm{PO}, \mathrm{PV}^{*} \quad 2 \quad 0 / 5$

PO, Y, PV $60 \quad 0 / 173$

PO, Y, PV $19 \quad 0 / 53$

$\begin{array}{lll}\mathrm{Y}, \mathrm{PV} & 5 & 1 / 17\end{array}$

$\begin{array}{lll}\text { PV } & 5 & 1 / 9\end{array}$

PO, Y, PV $11 \quad 3 / 20$

PO, Y, PV $21 \quad 2 / 44$

CR

$\begin{array}{lll}\mathrm{PV}^{*} & 1 & 0 / 1\end{array}$

PO, Y, PV 79 6/203
PO, Y, PV $6 \quad 0 / 10$

PO: Atheta fungi (2), Atheta paracrassicornis (1), Atheta pilicornis (1), Atheta subtilis (1), § (2), Tachinus laticollis (1), Epuraea variegata (1), Rhizophagus dispar (8), Dorcatoma dresdensis (2), Atomaria affinis (1) Y: Cis quadridens (1), Dolichocis laricinus (1) PO \& Y: Cis bidentatus (4)

PO: Dinaraea aequata (1), Rhizophagus dispar (1)

PV: Scaphisoma subalpinum (1), Atheta picipes (1), Corticaria lapponica (4)

PV: Cis glabratus (10+)

Y: Cis dentatus $\dagger$

PV: Acrulia inflata (1)

PV: Scaphisoma inopinatum (2)

PO: Orchesia fasciata (1) Y: Cis comptus (10+) Y \& PV: Cis bidentatus (3), Dolichocis laricinus (9)

PO: Gyrophaena affinis (1) PV: Cis bidentatus (10+), Orthoperus rogeri (1)

PO: Oxypoda hansseni (1) PV: Orchesia micans (5), Lordithon speciosus (2), Bolitochara pulchra (11), Atheta picipes (1), Atheta boletophila (8), Rhizophagus dispar (2), Dolichocis laricinus (1) PO \& PV: Abdera affinis (15)

$\begin{array}{lll}\text { PV } & 1 & 0 / 1\end{array}$

Y, PV $12 \quad 5 / 21$

PO, Y, PV $\quad 30 \quad 2 / 47$

Y, PV $\quad 11 \quad 2 / 43$

Y, PV $20 / 3$

Y, PV $\quad 31 \quad 2 / 72$

$\begin{array}{lll}\mathrm{Y}, \mathrm{PV}^{*} & 2 & 0 / 2\end{array}$
PV: Abdera affinis (3), Abdera flexuosa (2), Dorcatoma dresdensis (6), Orchesia micans (4)

PV: Cis bidentatus (2), Cis jacquemartii (1)

PV: Sciophila buxtoni (1 male)

61 Junghuhnia luteoalba (P. Karst.) Ryvarden

62 Lenzites betulinus (L. : Fr.) Fr. 
63 Leptoporus mollis (Pers. : Fr.) Quél.

64 Meruliopsis taxicola (Pers. : Fr.) Bondartsev

65 Oligoporus rennyi (Berk. \& Broome) Donk

66 Oligoporus sericeomollis (Romell) M. Bondartseva

67 Onnia leporina (Fr.) H. Jahn

68 Perenniporia subacida (Peck) Donk

69 Phellinus chrysoloma (Fr.) Donk

70 Phellinus conchatus (Pers. : Fr.) Quél.

71 Phellinus ferrugineofuscus (P. Karst.) Bourdot

72 Phellinus igniarius (L. : Fr.) Quél.

73 Phellinus laevigatus (P. Karst.) Bourdot \& Galzin

74 Phellinus lundellii Niemelä

75 Phellinus nigrolimitatus (Romell) Bourdot \& Galzin

76 Phellinus pini (Brot. : Fr.) A. Ames

77 Phellinus populicola Niemelä

78 Phellinus punctatus (P. Karst.) Pilát

79 Phellinus tremulae (Bondartsev)

Bondartsev \& Borisov

80 Phellinus viticola (Schwein. ex Fr.) Donk

81 Physisporinus vitreus (Pers. : Fr.) P. Karst.

82 Piptoporus betulinus (Bull. : Fr.) P. Karst.

83 Polyporus brumalis (Pers. : Fr.) Fr.

84 Polyporus ciliatus Fr. : Fr.

85 Polyporus leptocephalus (Jacq. : Fr.) Fr.

86 Polyporus melanopus (Pers. : Fr.) Fr

87 Polyporus pseudobetulinus (Pilát) Thorn, EN Kotir. \& Niemelä

88 Polyporus tubaeformis (P. Karst.) Ryvarden \& Gilb.

89 Porpomyces mucidus (Pers. : Fr.) Jülich

90 Postia alni Niemelä \& Vampola

91 Postia balsamina Niemelä \& Y.C. Dai

92 Postia caesia (Schrad. : Fr.) P. Karst.

93 Postia fragilis (Fr.) Jülich

94 Postia guttulata (Peck) Jülich

95 Postia parva (Renvall) comb. ined.

96 Postia lateritia Renvall

97 Postia leucomallella (Murrill) Jülich

98 Postia persicina Niemelä \& Y.C. Dai

99 Postia hibernica (Berk. \& Broome) Jülich sensu typi

100 Postia stiptica (Pers. : Fr.) Jülich

101 Postia tephroleuca (Fr.) Jülich

102 Protomerulius caryae (Schwein.) Ryvarden
PO, Y, PV $16 \quad 5 / 37$

PO, Y, PV $14 \quad 0 / 79$

$\begin{array}{lll}\mathrm{Y}, \mathrm{PV} & 4 & 0 / 6\end{array}$

PO, Y, PV $53 \quad 1 / 71$

PO, Y, PV $10 \quad 2 / 74$

$\begin{array}{llll}\text { NT } & \text { Y, PV } & 9 & 0 / 21\end{array}$

PO, Y, PV $56 \quad 1 / 168$

$\mathrm{PO}, \mathrm{Y}, \mathrm{PV} \quad 45 \quad 3 / 93$

\begin{tabular}{|c|c|c|c|}
\hline \multirow[t]{3}{*}{ NT } & $\mathrm{PO}, \mathrm{Y}, \mathrm{PV}$ & 48 & $0 / 143$ \\
\hline & $\mathrm{PO}, \mathrm{Y}, \mathrm{PV}$ & 71 & $1 / 224$ \\
\hline & $\mathrm{PO}, \mathrm{Y}, \mathrm{PV}$ & 45 & $0 / 106$ \\
\hline & $\mathrm{PO}, \mathrm{Y}, \mathrm{PV}$ & 25 & $1 / 72$ \\
\hline & $\mathrm{PO}, \mathrm{Y}, \mathrm{PV}$ & 51 & $0 / 16$ \\
\hline & $\mathrm{PO}, \mathrm{Y}, \mathrm{PV}$ & 26 & $0 / 29$ \\
\hline & $\mathrm{Y}, \mathrm{PV}$ & 20 & $2 / 28$ \\
\hline & PV & 1 & $0 / 1$ \\
\hline & $\mathrm{PO}, \mathrm{Y}, \mathrm{PV}$ & 68 & $0 / 112$ \\
\hline & $\mathrm{PO}, \mathrm{Y}, \mathrm{PV}$ & 92 & $0 / 2$ \\
\hline & $\mathrm{Y}, \mathrm{PV}^{*}$ & 2 & $0 / 6$ \\
\hline & $\mathrm{PO}, \mathrm{Y}, \mathrm{PV}$ & 45 & $0 / 156$ \\
\hline & $\mathrm{Y}, \mathrm{PV}$ & 6 & $0 / 12$ \\
\hline & PV & 8 & $0 / 8$ \\
\hline & $\mathrm{PO}, \mathrm{Y}, \mathrm{PV}$ & 16 & $2 / 42$ \\
\hline
\end{tabular}

$\begin{array}{lll}\text { PV } & 1 & 0 / 1\end{array}$

$\begin{array}{lll}\mathrm{PV} & 2 & 5 / 5\end{array}$

PV: Hallomenus axillaris (1), Bolitophila occlusa (2 males, 2 females), Sciaridae G.sp. (1)

PO: Cis hispidus (1)

PO: Lordithon speciosus (1), Rhizophagus sp. (1)

PO: Atheta crassicornis (1)

PO: Sulcacis fronticornis (1), Cis hispidus (1) PV: Cis bidentatus (10+), Abdera affinis (2), Abdera flexuosa (1), Orthoperus rogeri (1)

PV: Dorcatoma dresdensis (1)

PO: Cis dentatus (1)

PV: Dorcatoma dresdensis (1), Atomaria badia (1)

PO: Sulcacis affinis (1) PV: Cis jacquemartii (4), Cis bidentatus (3)

PV: Cis comptus (10+), Cis bidentatus (10+), Dacne bipustulata (10+), Atheta picipes (1), Cis boleti (1), Cerylon ferrugineum (2), Orthoperus rogeri (2), Orthoperus corticalis (1), Corticarina lambiana (1)

$\begin{array}{lll}\text { Y, PV } & 3 & 0 / 6 \\ \text { Y, PV } & 2 & 0 / 7 \\ \text { PO, Y, PV } & 15 & 1 / 34 \\ & & \\ \text { Y, PV } & 5 & 0 / 7 \\ \text { PO, Y, PV } & 16 & 0 / 47 \\ \text { Y, PV } & 4 & 0 / 14 \\ \text { PV } & 2 & 0 / 3 \\ \text { Y, PV } & 7 & 0 / 20 \\ \text { Y, PV } & 14 & 1 / 35 \\ \text { PV } & 16 & 1 / 40 \\ \text { Y, PV } & 2 & 0 / 2 \\ \text { Y, PV } & 2 & 0 / 3 \\ & & \\ \text { PV } & 2 & 0 / 9 \\ \text { PV } & 3 & 0 / 4 \\ \text { Y, PV } & 3 & 0 / 3\end{array}$

PV: Bolitophila occlusa (5 males 3 females)

Y: Cis dentatus $\dagger$

PV: Hallomenus axillaris (2) 
103 Pycnoporellus alboluteus (Ellis \& Everh.) EN Kotl. \& Pouzar

104 Pycnoporellus fulgens (Fr.) Donk

105 Pycnoporus cinnabarinus (Jacq. : Fr.)

P. Karst.

106 Rhodonia placenta (Fr.) Niemelä, K.H. NT Larsson \& Schigel

107 Rigidoporus corticola (Fr.) Pouzar

108 Rigidoporus populinus (Schumach. : Fr.)

Pouzar

109 Sistotrema alboluteum (Bourdot \& Galzin) NT Bondartsev \& Singer

110 Sistotrema muscicola (Pers.) S. Lundell

111 Skeletocutis amorpha (Fr.) Kotl. \& Pouzar

112 Skeletocutis biguttulata (Romell) Niemelä

113 Skeletocutis borealis Niemelä

114 Skeletocutis brevispora Niemelä

115 Skeletocutis carneogrisea A. David

116 Skeletocutis chrysella Niemelä

117 Skeletocutis jelicii Tortić \& A. David

118 Skeletocutis kuehneri A. David

119 Skeletocutis lilacina A. David \& Jean Kel- VU ler

120 Skeletocutis odora (Sacc.) Ginns

121 Skeletocutis papyracea A. David

122 Skeletocutis stellae (Pilát) Jean Keller

123 Spongiporus undosus (Peck) A. David

124 Trametes ochracea (Pers.) Gilb. \& Ryvarden

125 Trametes pubescens (Schumach. : Fr.) Pilát

126 Trametes velutina (Fr.) G. Cunn.

127 Trechispora hymenocystis (Berk. \& Broome) K.H. Larsson

128 Trechispora mollusca (Pers. : Fr.) Liberta

129 Trichaptum abietinum (Pers. : Fr.) Ryvarden

130 Trichaptum fuscoviolaceum (Ehrenb. : Fr.) Ryvarden

131 Trichaptum laricinum (P. Karst.) Ryvarden NT

132 Tyromyces chioneus (Fr.) P. Karst.

Non-polypore fungi

133 Amylostereum chailletii (Pers. : Fr.) Boidin

134 Asterodon ferruginosus Pat.

135 Bankera fuligineoalba (J. C. Schmidt : Fr.) Pouzar

136 Bankera violascens (Alb. \& Schw. : Fr.) Pouz.

137 Basidioradulum radula (Fr.) Nobles

138 Botryobasidium subcoronatum (Höhn. \& Litsch.) Donk

139 Byssomerulius albostramineus (Torrend) Hjortstam

140 Ceraceomyces borealis (Romell) J. Erikss. \& Ryvarden

141 Ceraceomyces serpens (Toode: Fr.) Ginns

142 Cantharellus cibarius Fr.

143 Cantharellus tubaeformis (Bull. : Fr.) Fr.

$\begin{array}{lll}\text { PV } & 4 & 0 / 33 \\ & & \\ \text { PV }^{*} & 1 & 0 / 1 \\ \text { PO, Y, PV* } & 3 & 0 / 4 \\ \text { PV } & 7 & 3 / 25\end{array}$

PV: Acrulia inflata (1), Gyrophaena strictula (1), Phymatura brevicollis (42), Scaphisoma inopinatum (1), Hallomenus axillaris (1)

PV: Cis bidentatus (1), Acrulia inflata (1)
NT

PV* $10 / 3$

$\begin{array}{lll}\mathrm{Y} & 2 & 0 / 2\end{array}$

$\begin{array}{lll}\mathrm{Y}, \mathrm{PV} & 5 & 0 / 8\end{array}$

PO, Y, PV $15 \quad 0 / 28$

$\begin{array}{lll}\mathrm{Y}, \mathrm{PV} & 7 & 0 / 12\end{array}$

$\begin{array}{llll}\text { EN } & Y & 4 & 0 / 6\end{array}$

$\begin{array}{llll}\text { VU } & \text { Y, PV } & 19 & 0 / 46\end{array}$

$\begin{array}{lll}\mathrm{Y} & 3 & 0 / 3\end{array}$

$\begin{array}{lll}Y, P V & 11 & 0 / 25\end{array}$

$\begin{array}{lll}\text { PV* } & 1 & 0 / 1\end{array}$

$\begin{array}{lll}\mathrm{Y}, \mathrm{PV} & 6 & 0 / 15\end{array}$

$\begin{array}{lll}\mathrm{PO}, \mathrm{Y} & 7 & 0 / 12\end{array}$

PO, Y, PV $17 \quad 3 / 98$

$\begin{array}{lll}\text { PV } & 3 & 0 / 9\end{array}$

$\begin{array}{lll}\text { Y, PV } & 16 & 0 / 48\end{array}$

$\begin{array}{lll}\mathrm{Y}, \mathrm{PV} & 5 & 0 / 16\end{array}$

PO, Y, PV $55 \quad 4 / 152$

PO, Y, PV $16 \quad 0 / 51$

PO, Y, PV $12 \quad 0 / 22$

PO, Y, PV $4 \quad 0 / 6$

PO, Y, PV $6 \quad 0 / 11$

PO, Y, PV $97 \quad 0 / 238$

PO, Y, PV $45 \quad 0 / 96$

PO, Y, PV $13 \quad 0 / 42$

$\mathrm{PO}, \mathrm{Y}, \mathrm{PV}^{*} 4 \quad 0 / 13$

PO: Leptusa pulchella (1) PV: Acrulia inflata (1), Orthoperus rogeri (5), Orthoperus corticalis (4)

PO: Acrulia inflata (1), Sulcacis fronticornis (1) Y: Cis hispidus (10+), Octotemnus glabriculus (2) PO \& Y: Cis boleti (10+)

$\begin{array}{lll}\text { Y } & 8 & 0 / 8 \\ \text { Y, PV } & 39 & 0 / 41 \\ \text { PO } & 1 & 0 / 1 \\ & & \\ \text { PO } & 1 & 0 / 1 \\ & & \\ \text { Y } & 1 & 0 / 1 \\ \text { Y } & 1 & 0 / 1 \\ & & \\ \text { Y } & 1 & 0 / 1 \\ \text { Y } & 1 & 0 / 1 \\ & & \\ \text { Y } & 1 & 0 / 1 \\ \text { Y } & 1 & 0 / 1 \\ \text { Y } & 1 & 0 / 1\end{array}$


144 Chaetodermella luna (Romell) Rausch.

145 Chondrostereum purpureum (Pers. : Fr.) Pouzar

146 Clavaria purpurea O.F. Müll. : Fr.

147 Clavicorona pyxidata (Pers. : Fr.) Doty

148 Columnocystis abietina (Pers. : Fr.) Pouzar

149 Conferticium ochraceum (Fr. : Fr.) Hallenb.

150 Coniophora olivacea (Pers. : Fr.) P. Karst.

151 Coniophora puteana (Schumacher: Fr.)

P. Karst.

152 Crustoderma dryinum (Berk. \& M.A.

Curtis) Parmasto

153 Cystostereum murrayi (Berk. \& M.A. Curtis) Pouzar

154 Gloiodon strigosus (Schwein. : Fr.) P. Karst.

155 Hericium cirrhatum (Pers.) Nikolajeva

156 Hericium coralloides (Scop. : Fr.) Pers.

157 Hydnellum caeruleum (Hornem.) P. Karst.

158 Hydnellum gracilipes (P. Karst.) P. Karst.

159 Hydnellum peckii Banker

160 Hydnum repandum L. : Fr.

161 Hydnum rufescens Schaeff. : Fr.

162 Hymenochaete fuliginosa (Pers.) Bres.

163 Hymenochaete tabacina (Fr.) Lév.

164 Hyphodontia aspera (Fr.) J. Erikss.

165 Kavinia alboviridis (Morgan) Gilb. \& Budington

166 Kuehneromyces mutabilis (Schaeff. : Fr.)

Singer \& Smith

167 Laeticorticium roseum (Fr.) Donk

168 Laurilia sulcata (Burt) Pouzar

169 Laxitextum bicolor (Pers. : Fr.) Lentz

170 Lentinellus vulpinus (Sowerby) Kuehner \& Maire

171 Leucogyrophana romellii (Fr.) Ginns

172 Lycogala flavofuscum (Ehrenb.) Rost

173 Lyophyllum ulmarium (Bull. : Fr.) Kühn.

174 Mucronella bresadolae (Quél.) Corner

175 Mucronella flava Corner

176 Mycoacia fuscoatra (Fr. : Fr) Donk

177 Odonticium romellii (S. Lundell) Parmasto

178 Phanerochaete laevis (Pers. : Fr.) J. Erikss. \& Ryvarden

179 Phanerochaete sanguinea (Fr.) Pouzar

180 Phanerochaete velutina (DC : Fr.) P. Karst.

181 Phellodon connatus (Schultz. : Fr.)

P. Karst.

182 Phellodon secretus Niemelä \& Kinnunen

183 Phellodon tomentosus (L. : Fr.) Banker

184 Phlebia centrifuga P. Karst.

185 Phlebia cornea (Bourd. \& Galzin) Parmasto

186 Phlebia gigantea Fr. : Fr.

187 Phlebia radiata Fr.

188 Phlebia segregata (Bourd. \& Galzin) Parmasto

189 Phlebia subulata J. Erikss. \& Hjortstam

190 Phlebia tremellosa (Schrad. : Fr.) Burds. \& Nakasone

191 Phlebiella vaga (Fr. : Fr.) P. Karst. (P. sulphurea)

192 Phyllotopsis nidulans (Pers. : Fr.) Singer

193 Piloderma fallax (Liberta) Stalpers

\begin{tabular}{|c|c|c|c|}
\hline & Y, PV & 43 & $0 / 43$ \\
\hline & & 9 & $0 / 9$ \\
\hline & $\mathrm{Y}$ & 1 & $0 / 1$ \\
\hline & PV & 4 & $0 / 4$ \\
\hline & $\mathrm{Y}, \mathrm{PV}$ & 64 & $0 / 82$ \\
\hline & Y & 1 & $0 / 1$ \\
\hline & $\mathrm{Y}, \mathrm{PV}$ & 7 & $0 / 7$ \\
\hline & Y & 1 & $0 / 1$ \\
\hline NT & $\mathrm{Y}$ & 1 & $0 / 1$ \\
\hline NT & Y, PV & 6 & $0 / 7$ \\
\hline & $\mathrm{Y}$ & 1 & $0 / 1$ \\
\hline & $\mathrm{PO}, \mathrm{Y}$ & 3 & $2 / 5$ \\
\hline & $\mathrm{Y}, \mathrm{PV}$ & 15 & $0 / 15$ \\
\hline & $\mathrm{PO}, \mathrm{Y}$ & 2 & $0 / 2$ \\
\hline & Y & 2 & $0 / 2$ \\
\hline & $\mathrm{PO}, \mathrm{Y}$ & 2 & $0 / 2$ \\
\hline & $\mathrm{Y}, \mathrm{PV}$ & 13 & $0 / 13$ \\
\hline & Y, PV & 3 & $0 / 3$ \\
\hline & Y & 3 & $0 / 3$ \\
\hline & $\mathrm{Y}$ & 1 & $0 / 1$ \\
\hline & Y & 1 & $0 / 1$ \\
\hline NT & $\mathrm{Y}, \mathrm{PV}$ & 2 & $0 / 2$ \\
\hline & $\mathrm{Y}$ & 1 & $0 / 1$ \\
\hline & $\mathrm{PO}, \mathrm{PV}$ & 2 & $0 / 2$ \\
\hline NT & $\mathrm{PO}, \mathrm{Y}$ & 33 & $0 / 41$ \\
\hline & Y, PV & 5 & $0 / 5$ \\
\hline & Y & 2 & $0 / 2$ \\
\hline & $\mathrm{Y}$ & 1 & $0 / 1$ \\
\hline & $\mathrm{Y}$ & 1 & $0 / 1$ \\
\hline & $\mathrm{Y}$ & 1 & $0 / 1$ \\
\hline & Y & 1 & $0 / 1$ \\
\hline & $\mathrm{Y}$ & 9 & $0 / 10$ \\
\hline & $\mathrm{Y}$ & 1 & $0 / 1$ \\
\hline NT & Y & 1 & $0 / 1$ \\
\hline & $\mathrm{Y}$ & 1 & $0 / 1$ \\
\hline & $\mathrm{Y}, \mathrm{PV}$ & 4 & $0 / 4$ \\
\hline & $\mathrm{Y}$ & 1 & $0 / 1$ \\
\hline & $\mathrm{Y}$ & 1 & $0 / 1$ \\
\hline & $\mathrm{Y}$ & 1 & $0 / 1$ \\
\hline & $\mathrm{PO}, \mathrm{Y}$ & 1 & $0 / 1$ \\
\hline VU & $\mathrm{PO}, \mathrm{Y}, \mathrm{PV}$ & 80 & $0 / 96$ \\
\hline NT & $\mathrm{Y}, \mathrm{PV}$ & 9 & $0 / 9$ \\
\hline & $\mathrm{Y}$ & 1 & $0 / 1$ \\
\hline & $\mathrm{PO}, \mathrm{Y}, \mathrm{PV}$ & 23 & $0 / 23$ \\
\hline & Y & 1 & $0 / 1$ \\
\hline & $\mathrm{Y}$ & 1 & $0 / 1$ \\
\hline & Y, PV & 27 & $0 / 27$ \\
\hline & $\mathrm{Y}$ & 3 & $0 / 3$ \\
\hline & $\mathrm{Y}, \mathrm{PV}$ & 6 & $0 / 6$ \\
\hline & $\mathrm{Y}$ & 1 & $0 / 1$ \\
\hline
\end{tabular}

PO: Cis dentatus (2), Mycetophagus multipunctatus (1) 
194 Pleurotus pulmonarius (Fr.) Quél.
PO, PV $22 / 2$

$\begin{array}{llll} & \text { Y, PV } & 12 & 0 / 13 \\ \text { Y, PV } & 6 & 0 / 6 \\ \text { PV } & 1 & 0 / 1 \\ \text { Y } & 1 & 0 / 1 \\ \text { Y } & 5 & 0 / 5 \\ \text { NT } & \text { Y } & 1 & 0 / 1 \\ & \text { Y, PV } & 8 & 0 / 8 \\ & \text { Y, PV } & 21 & 0 / 22 \\ \text { Y, PV } & 10 & 0 / 10 \\ \text { Y, PV } & 16 & 0 / 16 \\ & & \\ \text { Y } & 1 & 0 / 1 \\ \text { Y, PV } & 5 & 0 / 5 \\ \text { Y } & 1 & 0 / 1 \\ \text { Y } & 1 & 0 / 1 \\ \text { Y } & 1 & 0 / 1\end{array}$

PO: Lordithon trimaculatus (3), Lordithon lunulatus (2), Lordithon thoracicus (1), Atheta subtilis (4), Anopleta corvina (1), Atheta nigricornis (1), Omalium strigicolle (2), Tachyporus abdominalis (1), Tetratoma ancora (1), PV: Omalium rivulare (1), Deliphrum tectum (9), Atheta lapponica (4), Atheta aeneipennis (10), Proteinus brachypterus (1), Phymatura brevicollis (3), Gyrophaena fasciata (1), Cerylon ferrugineum (3), PO \& $\mathbf{P V}$ : Lordithon speciosus (6), Atheta picipes (9), Rhizophagus dispar (31), Triplax scutellaris (7)
195 Plicatura nivea (Sommerf. : Fr.) P. Karst.

196 Pseudomerulius aureus (Fr.) Jülich

197 Ramaria flava (Schaeff. : Fr.) Quél.

198 Sarcodon imbricatus (L. : Fr.) P. Karst.

199 Serpula himantioides (Fr. : Fr.) P. Karst.

200 Sistotrema confluens Pers. : Fr.

201 Sistotrema raduloides (P. Karst.) Donk

202 Stereum hirsutum (Willd. : Fr.) Gray

203 Stereum rugosum Pers. : Fr.

204 Stereum sanguinolentum (Alb. \& Schwein. : Fr.) Fr.

205 Stropharia hornemannii (Fr. : Fr.) Lundell

206 Thelephora terrestris Ehrh. : Fr.

207 Tomentella bryophila (Pers.) M.J. Larsen

208 Tubaria confragosa (Fr.) Kühn.

209 Vibrissea truncorum Fr.
Table 3. Systematic list of beetles attracted to polypores in western Finnish Lapland. FS/IUCN = Frequency score of beetles (Rassi 1993) / threat categories (Rassi et al. 2001); threat categories and scores of the uncommon and rare beetle species in Finland (30 points and higher) are indicated in bold face; $\mathrm{L}=$ locality: Y $=$ Ylläs-Aakenus National Park, PV = Pisavaara Strict Nature Reserve, PO = Pallas-Ounastunturi National Park; bold face $=$ new for the area Literature data on localities are marked as following: $+=$ Lindberg $\&$ Saris (1952), 粰 $=$ Wegelius (1960), $\diamond=$ Sippola et al. (1995), $\dagger=$ Rintala et al. (2003). Host fungi: Numbers refer to the species of fungi (Table 2); light face = records of adult beetles; bold face $=$ larvae or rearings.

\begin{tabular}{|c|c|c|c|}
\hline Taxon & FS/IUCN & $\mathbf{L}$ & Host fungi \\
\hline \multicolumn{4}{|l|}{ COLEOPTERA } \\
\hline \multicolumn{4}{|l|}{ Staphylinidae Latreille, 1802} \\
\hline Acrulia inflata (Gyllenhal, 1813) & 6 & $\mathrm{PO}, \mathrm{Y} \dagger, \mathrm{PV}$ & $3,19,49,106,107,120,124$ \\
\hline Anopleta corvina (Thomson, 1856) & 15 & $\mathrm{PO}$ & 194 \\
\hline Atheta aeneipennis (Thomson, 1856) & 2 & $\mathrm{PO} \diamond, \mathrm{PV}, \mathrm{Y} \dagger$ & 194 \\
\hline Atheta boletophila (Thomson, 1856) & 30 & PV & 55 \\
\hline Atheta crassicornis (Fabricius, 1793) & 4 & PO, $\mathrm{Y} \dagger, \mathrm{PV} \ddagger$ & 69 \\
\hline Atheta fungi (Gravenhorst, 1806) & 1 & $\mathrm{PO}, \mathrm{Y} \dagger, \mathrm{PV} \ddagger$ & 33 \\
\hline Atheta lapponica J. Sahlberg, 1876 & 20 & PV & 194 \\
\hline Atheta paracrassicornis Brundin, 1954 & 2 & PO & 33 \\
\hline Atheta nigricornis (Thomson, 1852) & 6 & $\mathrm{PO}, \mathrm{Y} \dagger$ & 194 \\
\hline Atheta picipes (Thomson, 1856) & 15 & $\mathrm{PO} \diamond, \mathrm{Y} \dagger, \mathrm{PV}$ & $40,55,87,194$ \\
\hline Atheta pilicornis (Thomson, 1852) & 10 & $\mathrm{PO}, \mathrm{PV} \ddagger$ & 33 \\
\hline Atheta subtilis (Scriba, 1866) & 1 & $\mathrm{PO}, \mathrm{Y} \dagger$ & 33,194 \\
\hline Bolitochara pulchra (Gravenhorst, 1806) & 2 & $\mathrm{Y} \dagger, \mathrm{PV}$ & 55 \\
\hline
\end{tabular}


Deliphrum tectum (Paykull, 1789)

Dinaraea aequata (Erichson, 1837)

Gyrophaena affinis Mannerheim, 1830

Gyrophaena fasciata (Marsham, 1802)

Gyrophaena strictula Erichson, 1839

Leptusa pulchella (Mannerheim, 1830)

Lordithon lunulatus (Linnaeus, 1761)

Lordithon speciosus (Erichson, 1840)

Lordithon thoracicus (Fabricius, 1777)

Lordithon trimaculatus (Fabricius, 1793)

Omalium rivulare (Paykull, 1789)

Omalium strigicolle Wankowicz, 1869

Oxypoda hansseni Strand, 1946

Quedius plagiatus Mannerheim, 1843

Phyllodrepa linearis (Zetterstedt, 1828)

Phymatura brevicollis (Kraatz, 1856)

Proteinus brachypterus (Fabricius, 1792)

Scaphisoma inopinatum Löbl, 1967

Scaphisoma subalpinum Reitter, 1881

Sepedophilus littoreus (Linnaeus, 1758)

Tachinus laticollis Gravenhorst, 1802

Tachyporus abdominalis (Fabricius, 1781)

Anobiidae Fleming, 1821

Dorcatoma dresdensis Herbst, 1792

Trogossitidae Latreille, 1802

Ostoma ferruginea (Linnaeus, 1758)

Nitidulidae Latreille, 1802

Epuraea variegata (Herbst, 1793)

Monotomidae Laporte de Castelnau, 1840

Rhizophagus dispar (Paykull, 1800)

Rhizophagus sp.

Cryptophagidae Latreille, 1802

Atomaria affinis (F. Sahlberg, 1834)

Atomaria badia Erichson, 1846

Erotylidae Latreille, 1802

Dacne bipustulata (Thunberg, 1781)

Triplax scutellaris Charpentier, 1825

Cerylonidae Billberg, 1820

Cerylon ferrugineum Stephens, 1830

Corylophidae LeConte, 1852

Orthoperus corticalis (Redtenbacher, 1849)

Orthoperus rogeri Kraatz, 1874

Latridiidae Erichson, 1842

Corticaria lapponica (Zetterstedt, 1838)

Corticaria longicollis (Zetterstedt, 1838)

Corticarina lambiana (Sharp, 1910)

Ciidae Leach, 1819

Cis bidentatus (Olivier, 1790)

Cis boleti (Scopoli, 1763)

Cis comptus Gyllenhal, 1827

Cis dentatus Mellié, 1848

Cis glabratus Mellié, 1848

Cis hispidus (Paykull, 1798)

Cis micans (Fabricius, 1792)

Cis jacquemartii Mellié, 1848

Cis quadridens Mellié, 1848

Dolichocis laricinus (Mellié, 1848)

Octotemnus glabriculus (Gyllenhal, 1827)

Sulcacis affinis (Gyllenhal, 1827)

\section{1}

2

2

2

15

1

2

20

\section{1}

30

1

15

100

2

15

60

\section{1}

10

6

4

2

10

15

10

6

2

30

40

6

15

4

15

15

2

40

20

1

10

40

6

2

NT/20

2

60

20

10

1
$\mathrm{PO} \diamond, \mathrm{Y} \dagger, \mathrm{PV} \quad 194$

$\mathrm{PO}, \mathrm{Y} \dagger \quad 36$

PO 53

PV 194

PV 106

$\mathrm{PO}, \mathrm{Y} \dagger, \mathrm{PV} \quad 19,33,120$

$\mathrm{PO}, \mathrm{Y} \dagger, \mathrm{PV} \neq \quad 194$

$\mathrm{PO}, \mathrm{Y} \dagger, \mathrm{PV} \quad 55,67,194$

$\mathrm{PO}, \mathrm{Y} \dagger, \mathrm{PV} \ddagger \quad 194$

PO, Y† 194

$\mathrm{PO}$ 祣, $\mathrm{Y} \dagger, \mathrm{PV} 194$

$\mathrm{PO}, \mathrm{Y} \dagger, \mathrm{PV} \ddagger \quad 194$

PO 55

$\mathrm{Y} \dagger, \mathrm{PV} \quad 3$

$\mathrm{PO} \diamond, \mathrm{Y} \dagger, \mathrm{PV} \quad 3,8$

PV 106, 194

$\mathrm{Y \dagger}, \mathbf{P V} \quad 194$

$\mathrm{Y} \dagger, \mathrm{PV} \quad 50,106$

$\mathrm{PO}$ 䄽, $\mathrm{Y} \dagger, \mathrm{PV} \quad 40$

$\mathrm{Y} \dagger, \mathrm{PV} \quad 3$

$\mathrm{PO}, \mathrm{Y} \dagger, \mathrm{PV} \ddagger \quad 33$

$\mathrm{PO}, \mathrm{Y} \dagger \quad 194$

PO, Y†, PV $\quad 33, \mathbf{5 7}, 72,77$

$\mathrm{PO}$ 粼, $\mathrm{Y} \dagger, \mathrm{PV} \quad 3$

$\mathrm{PO}, \mathrm{Y \dagger}, \mathrm{PV} \ddagger \quad 33$

$\mathrm{PO}, \mathrm{Y} \dagger, \mathrm{PV} \quad 19,33,36,56,194$

$\mathrm{PO}$

$\begin{array}{ll}\text { PO, Y, PV } & 3,33 \\ \text { PV } & 77\end{array}$

PO䄽, PV 85

$\mathrm{PO}, \mathrm{Y} \dagger, \mathrm{PV} \quad 194$

PO解, $\mathrm{Y} \dagger, \mathrm{PV} \quad 85,194$

PV $\quad 85,120$

PO, PV $\quad 3,14,53,70,87,120$

$\mathrm{PO}$ 解, $\mathrm{Y} \dagger, \mathrm{PV} \quad 40$

$\mathrm{PO}$ 愁, $\mathrm{PV} \quad 3$

PV 85

PO, Y, PV $\quad \mathbf{3 3}, \mathbf{5 2}, \mathbf{5 3}, \mathbf{5 8}, \mathbf{7 0}, \mathbf{8 5}, \mathbf{8 7}, 107$

PO, Y, PV $\quad 85, \mathbf{1 2 4}$

PO敖, Y, PV $\mathbf{5 2 , 8 7}$

PO, Y†, PV $\quad 12 \dagger, 42,74,96 \dagger, 155$

$\mathrm{Y} \dagger, \mathrm{PV}$

PO, $\mathrm{Y} \dagger, \mathrm{PV} \quad 66,70,124$

$\mathrm{Y} \dagger, \mathrm{PV} \quad 8$

$\mathrm{PO}$ 解, $\mathrm{Y} \dagger$, $\mathrm{PV} \quad \mathbf{1 9}, \mathbf{5 8 , 8 5}$

PO, PV

PO, $\mathrm{Y} \dagger, \mathrm{PV} \quad 33, \mathbf{5 2}, 55$

PV $\quad 124$

PO 85 
Sulcacis fronticornis (Panzer, 1809)

Mycetophagidae Leach, 1815

Mycetophagus multipunctatus Fabricius, 1792

Tetratomidae Billberg, 1820

Tetratoma ancora Fabricius, 1790

Melandryidae Leach, 1815

Hallomenus axillaris (Illiger, 1807)

Hallomenus binotatus (Quensel, 1790)

Abdera affinis (Paykull, 1799)

Abdera flexuosa (Paykull, 1799)

Orchesia fasciata (Illiger, 1798)

Orchesia micans (Panzer, 1794)

Curculionidae Latreille, 1802

Rhyncolus ater (Linnaeus, 1758)

\section{DIPTERA}

Sciaridae Edwards, 1925

Genus sp. indet.

Mycetophilidae Edwards, 1925

Bolitophila occlusa Edwards, 1913

Sciophila buxtoni Freeman, 1956
PO

$\mathrm{PO}, \mathrm{Y} \dagger$

$\mathrm{PO}, \mathrm{Y} \uparrow, \mathrm{PV} \ddagger$

PV

PV

PO, PV

$\mathrm{PO}$ 数, $\mathrm{Y} \dagger, \mathrm{PV}$

$\mathrm{PO}$

$\mathrm{PO}$ 解, $\mathrm{Y} \dagger, \mathrm{PV}$

$\mathrm{Y} \dagger, \mathrm{PV}$

PV

PV

PV
3,63

$3,12,70,124$

155

194

$\mathbf{6 3 , 9 7 ,} 106$

3

55, 57, 70

57, 70

52

55, 57

3

63,90

19, 61

\section{Results}

\subsection{Polypores}

A remarkably high number of 132 polypore species that we report here from western Finnish Lapland is confirming the irreplaceable nature values of this wilderness in northern Fennoscandia. We collected 128 species and four more are reported from herbaria (Table 2). Two species (Postia persicina, $P$. balsamina) were described as new to science (Niemelä et al. 2004). Several tens of rare species were collected, including 39 (25.9\%) red-listed (Rassi et al. 2001). The threatened species belong to different IUCN threat categories, including one CR-listed, Inonotopsis subiculosa. Of the six EN-listed species, Antrodia crassa, Polyporus pseudobetulinus, Pycnoporellus alboluteus, and Skeletocutis borealis were collected by us, while Skeletocutis jelicii, and Antrodiella canadensis derive from herbarium data. Twelve species of polypores are VU-listed and 20 NT-listed, as shown in Table 2, which totals 209 polypore and non-polypore species of fungi, but the latter were recorded and collected only inconsistently when time allowed. Of the non-polypore fungi the hydnaceous Phellodon secretus was described as a new species (Niemelä et al. 2003b).

\subsection{Beetles}

We collected 72 fungus-associated species of Coleoptera on 34 (25.8\% of studied) polypore species. A total of 34 insect species reported here are new to the studied area: 15 Coleoptera species were newly collected in the Pallas-Ounastunturi National Park, one is new to Ylläs-Aakenus, and 18 are new to Pisavaara Strict Nature Reserve, including one NT species (Cis micans). Twelve beetle species are rated with frequency scores (Rassi 1993) of 30 or higher, including one 100point staphylinid species (Oxypoda hansseni). Many beetle species were collected from previously unreported fungal substrates (Table 3).

\section{Discussion}

\subsection{Polypores and other fungi}

Inonotopsis subiculosa is one of the rarest basidiomycetes in the Old World. Its first European find was made in Pisavaara by Eriksson and Strid (1969) in a moist slope with old and dense spruce forest and ample coarse woody debris. In spite of repeated efforts during subsequent decades the 
species has never been found there again. Now it is known in North Europe from a few places in Sweden, and one in Norway (Tom Hellik Hofton, pers. comm.), Estonia (in 1965, not afterwards: Parmasto 2004), and Komi Republic in northern Russia (Parmasto 1973). It was considered extinct in Finland, until in 2005 it was collected anew and photographed in Central Finland (by Panu Halme, University of Jyväskylä, see Niemelä 2005).

All the EN-listed species - Antrodia crassa, Antrodiella canadensis, Polyporus pseudobetulinus, Pycnoporellus alboluteus, Skeletocutis borealis and $S$. jelicii - are rarities which need exceptionally well preserved old-growth forests for their survival. The four first-mentioned ones are confined to very large tree trunks, while the two Skeletocutis species usually grow on fairly thin trees or on decayed woody fragments. Of them the least well known may be $S$. borealis, described by Niemelä (1998); it was found in our area on brookside Prunus padus (Padus racemosa) thickets. In the Kainuu province of Finland, closer to the Russian border, it has been collected recently on shrubby Sorbus aucuparia, and in favourable brook- and riverside sites it can be locally common (Pekka Helo \& Teppo Helo, pers. comm.).

The twelve VU species are all characteristic polypores of old-growth forests. Some of them favour thick, fallen, still-hard trunks of spruce, like Amylocystis lapponica. Others are characteristic inhabitants of long-ago fallen pine trunks, often kelo trees (see Niemelä et al. 2002), like Antrodia infirma, A. primaeva, Postia lateritia and Skeletocutis stellae. Many of these occasionally grow also on spruce. Antrodiella citrinella is a strict successor species of Fomitopsis pinicola, and lives on spruce, less often on pine, birch, aspen and alder, provided that the preceding species was there. Skeletocutis lilacina is a successor of Trichaptum species, and almost all of its finds were made from moist spruce-dominated forests, for instance foothill seepages or wet brooksides.

Also all the NT species are rare ones, and indicate high protection values of their growth sites. Many of them are solely or predominantly northern in their distribution, if whole Europe is considered. The best examples are Gloeophyllum

Table 4. Construction of the fruit body and characteristic taxa of associated Coleoptera in northern and eastern Europe. Construction elements are illustrated in Figs. 4-6.

\begin{tabular}{|c|c|c|c|c|}
\hline Code & Construction element & Definition & Polypore species & $\begin{array}{l}\text { Typical taxa of } \\
\text { Coleoptera }\end{array}$ \\
\hline BF & Back face & $\begin{array}{l}\text { Contact area between the } \\
\text { fruit body and the woody } \\
\text { substrate }\end{array}$ & All & $\begin{array}{l}\text { Larvae of Trogossitidae } \\
\text { and Elateridae }\end{array}$ \\
\hline $\mathbf{C E}$ & Core & $\begin{array}{l}\text { Hard inner ball-like } \\
\text { structure inside the } \\
\text { basidiocarp, next to BF }\end{array}$ & $\begin{array}{l}\text { Several, annual } \\
\text { or perennial }\end{array}$ & Unknown \\
\hline CT & Crust & $\begin{array}{l}\text { Upper surface cover, } \\
\text { structurally different from } \\
\text { the underlying } \mathrm{C}\end{array}$ & $\begin{array}{l}\text { Several, annual } \\
\text { or perennial }\end{array}$ & $\begin{array}{l}\text { Unknown, Anobiidae, } \\
\text { and sometimes Ciidae are } \\
\text { hatching through crust }\end{array}$ \\
\hline $\mathbf{C}$ & $\begin{array}{l}\text { Context } \\
\text { (incl. subiculum) }\end{array}$ & $\begin{array}{l}\text { Mycelial layer above the } \\
\text { hymenophore }\end{array}$ & All & Cis, Sulcacis, Octotemnus \\
\hline $\mathbf{T}$ & $\begin{array}{l}\text { Context-hymenophore } \\
\text { transition }\end{array}$ & $\begin{array}{l}\text { Contact zone linking con- } \\
\text { text and hymenophore }\end{array}$ & All & Ennearthron \\
\hline TT & Tube-layer transitions & $\begin{array}{l}\text { Transitional mycelium } \\
\text { between yearly layers of } \\
\text { tubes }\end{array}$ & Perennial & Cis nitidus \\
\hline $\mathbf{H}$ & Hymenophore & Whole tube layer & All & Dorcatominae \\
\hline HS & Hymenophore surface & $\begin{array}{l}\text { Poroid (rarely gilled), } \\
\text { down-facing surface of } \\
\text { the basidiocarp }\end{array}$ & All & Adults of Trogossitidae \\
\hline $\mathbf{S}$ & Spores & $\begin{array}{l}\text { Mass of the spores, } \\
\text { airborne and then settled, } \\
\text { covering the fruit body } \\
\text { and surfaces around }\end{array}$ & $\begin{array}{l}\text { All, during the } \\
\text { sporulation } \\
\text { period }\end{array}$ & $\begin{array}{l}\text { Adults of Latridiidae, } \\
\text { Cerylonidae, Leiodidae }\end{array}$ \\
\hline
\end{tabular}


protractum, Haploporus odorus and Trichaptum laricinum. The others have a northern tendency, too, but are seldom found in the coniferous forests of high mountains in Central Europe, and even in the Balkans.

In addition, notes were made of the other fungi, mostly growing on wood. Among them we found a number of red-listed species: Crustoderma dryinum (NT), Cystostereum murrayi (NT), Kavinia alboviridis (NT), Laurilia sulcata (NT), Odonticium romellii (NT), Phlebia centrifuga (VU), Phlebia cornea (NT), and Sistotrema raduloides (NT). Both Hydnellum gracilipes and Phellodon secretus are extremely rare inhabitants of pristine pine forests, but their red list categories have not yet been assessed.

\subsection{Polypore fruit body as a habitat of Coleoptera}

In studies of polypore-associated beetle fauna, a fungal fruit body (basidiocarp, basidioma) was traditionally taken as a uniform unit, being consumed either by insect larvae or adult insects. The existence of multi-species associations within the basidiocarps of numerous polypore species reflects the structural (and probably nutrient and microclimatic) differences among different parts of a fungal fruit body. Insect preferences to various parts of the Fomes fomentarius pileus were described by Matthewman \& Pielou (1971).

In early myco-entomological studies the word "fungus" was commonly referred just to a fungal fruit body (basidiocarp), i.e. the reproductive part only. However, the whole organism includes mycelium together with its fruit bodies, and thus there may be several basidiocarps of a single fungal individual on a tree trunk, or several fruiting bodies of several individuals of one species (Rayner \& Boddy 1988). Also even-aged fungal fruit bodies display considerable differences in their beetle species compositions and in the number of beetle individuals, even when a single trunk is studied. These substrates are much more complex and heterogeneous than what they externally seem to be, and even neighbouring basidiomata of the same species (same individual?) differ in their sporulation periods (Parmasto \& Parmasto 2003). The moment of sporulation is an important turning-point, because then the basidiocarp is fully mature, and the growth of the basidiocarp (in annual species) or hymenophore's layer (in perennial species) is about to end.

Different parts of a fungus individual differ greatly from each other. While the basidiocarps are made of fairly solid hyphal tissue, the mycelium spreads out within wood in a cobweb-like fashion. From a beetle's point of view, these different parts show notable differences in construction, microclimatic conditions and nutrition value. Two major groups are separated of fungus-feeding Coleoptera: mycelium consumers and fruit body consumers. In our study we mostly deal with the latter, as understood in a wide sense, i.e. including spore feeders, and those attracted by anamorphic fungi and myxomycetes that grow over decomposed polypores.

The basic structure of polypore basidiocarp was described in introduction sections of numerous identification books (e.g. Ryvarden \& Gilbertson 1993), so here we give only a short summary of fruit body construction. Basidiocarp of a polypore fungus is a compact hyphal formation projecting from the substrate. The shape varies from pileate or stipitate to nodulose and resupinate. Volume and water contents also differ among the basidiocarps of different species and their varying age. Context layer, located between upper surface and hymenophore of the fruit body, may be homogeneous or layered. Context may be covered by crust, trichoderm or pellicle. Some perennial basidiocarps have a robust core located close to the woody substrate. The hymenophore, comprised of seasonal tube layers in perennial species, produces basidiospores leaving the ripe fruit body in suitable weather conditions. The shape and construction of the basidiocarp, and pore size of the hymenophore vary according to the species, which limits the size of beetles residing in tubes: Epuraea spp. can inhabit the wide tubes of Daedaleopsis confragosa, and Phloeocharis subtilissima dwells in Phellinus pini (Schigel et al. 2004), while only Baranowskiella ehnstromi is tiny enough to enter the narrow tubes of Phellinus conchatus (Sörensson 1997). If compared to agarics and most other fungal groups, polypores differ in having sturdy basidiocarps. Favoured by Coleoptera, they are perennial in many species, but even if annual, they are usually more persistent than e.g. mushrooms, dominated by Diptera.

The complex structure of the fungal fruit body (Figs. 4-6) offers both space and feeding substrate for a variety of larvae and adult insects. 


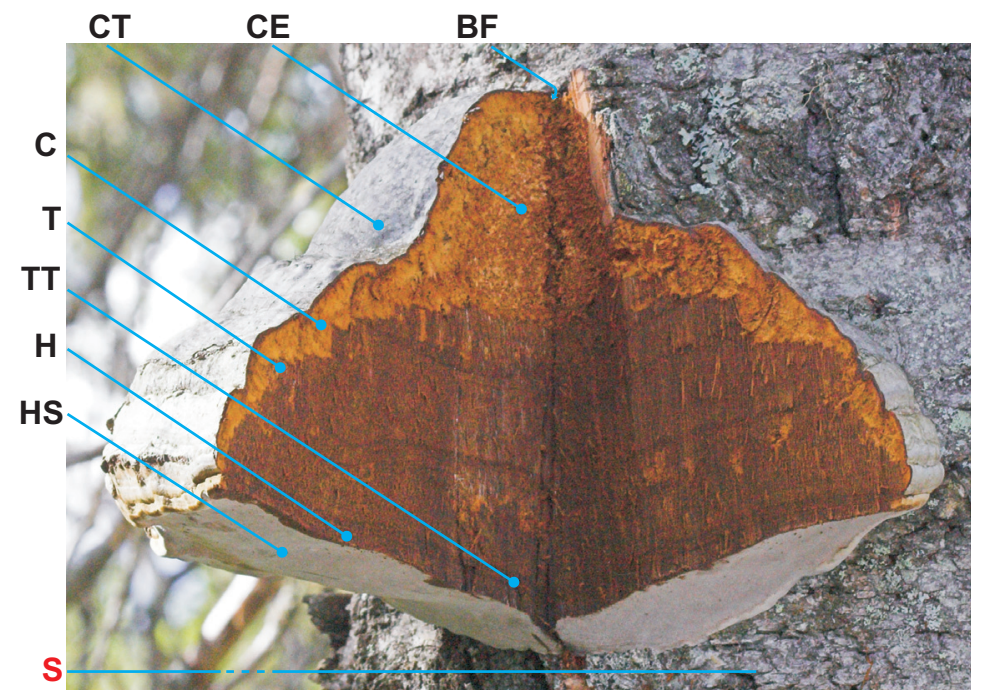

Fig. 4. Construction of the perennial fruit body of Fomes fomentarius, decomposition stage 1 , approx. one third of the fruit body removed. Construction elements (Table 4) are abbreviated as following: $\mathbf{B F}=$ back face, $\mathbf{C E}=$ core, $\mathbf{C T}=$ crust, $\mathbf{C}$ = context, $\mathbf{T}=$ context - hymenophore transition, TT $=$ tube-layer transitions, $\mathbf{H}=$ hymenophore, $\mathbf{H S}=$ hymenophore surface, $\mathbf{S}=$ spores. Photo DSS.

Fig. 5. Radial sections through the polypore fruit bodies (decomposition stage 1) of six consistency classes: PIP = piptoporoid, $\mathbf{F O M}$ = fomitoid, $\mathbf{X A N}=$ xanthochroic, TYR = tyromycetoid, TRA = trametoid, TRI = trichaptoid. Construction elements (Table 4) are abbreviated as following: $\mathrm{BF}=$ back face, $\mathrm{CE}=$ core, $\mathrm{CT}=$ crust, $\mathrm{C}=$ context, $\mathrm{T}=$ context-hymenophore transition, TT = tube-layer transitions, $\mathrm{H}=$ hymenophore, HS = hymenophore surface. Photo DSS.
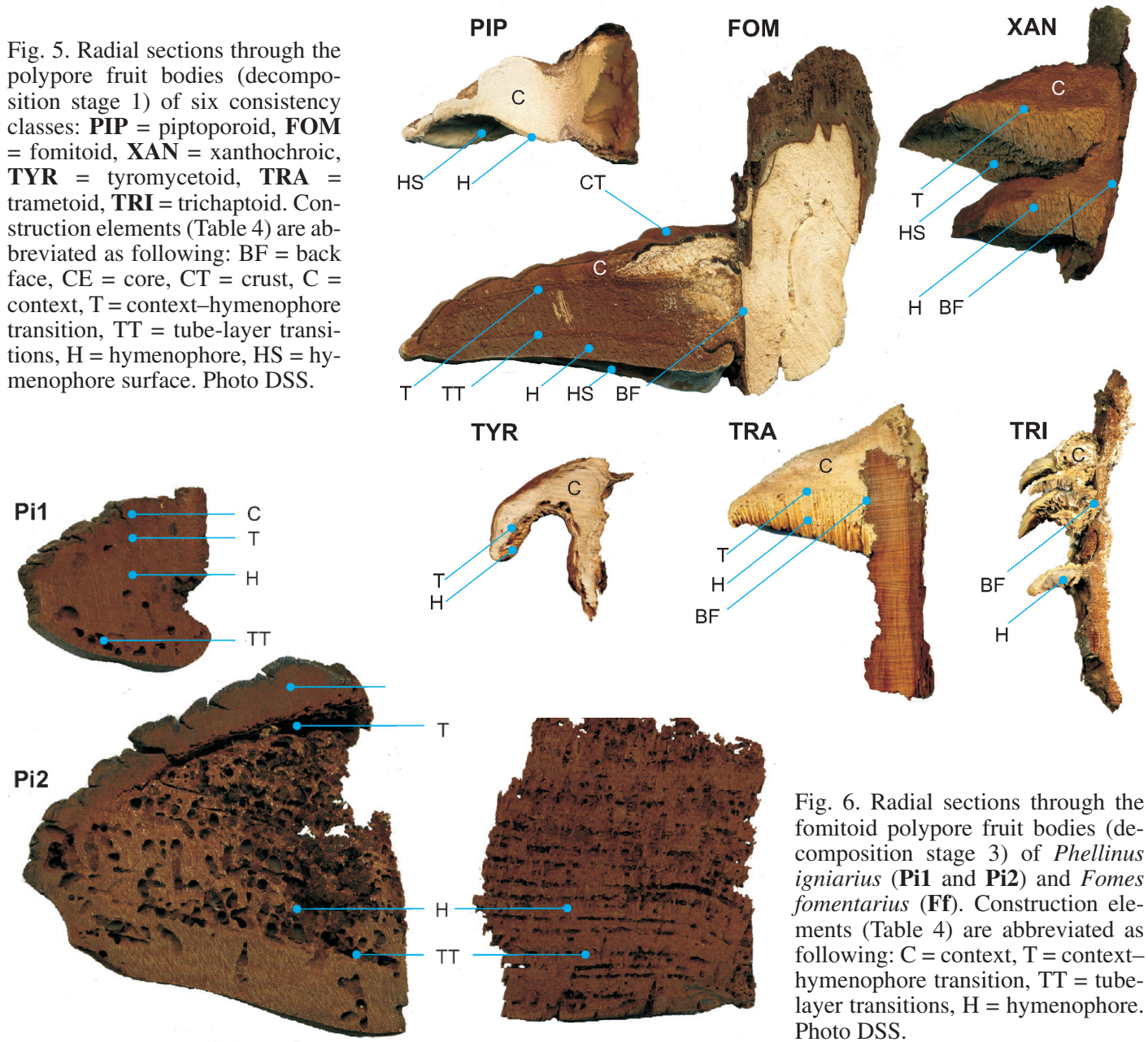

Fig. 6. Radial sections through the fomitoid polypore fruit bodies (decomposition stage 3 ) of Phellinus igniarius (Pi1 and Pi2) and Fomes fomentarius $(\mathbf{F f})$. Construction elements (Table 4) are abbreviated as following: $\mathrm{C}=$ context, $\mathrm{T}=$ context hymenophore transition, TT = tubelayer transitions, $\mathrm{H}=$ hymenophore. Photo DSS. 
The construction of polypore basidiocarp is illustrated on Fig. 4, and the terminology in principle follows Matthewman \& Pielou (1971) with minor changes (Table 4, Fig. 4). Variations of the construction in six consistency classes of polypores (Schigel et al. 2004) are shown on Figs. 5 and 6. The parts of fruit body that are preferred by beetles are more clearly seen in living or recently dead basidiocarps, but at the later stages of decomposition sapromycetophagous beetle larvae are less selective. Most larvae prefer context and context-hymenophore transition, but avoid robust core (Fig. 5). The majority of the Ciidae which colonize perennial fruit bodies favour the context-hymenophore transition and tube-layer transitions (Fig. 6).

Insect individuals of several species may coinhabit the fruit bodies and share spatial and ecological niches, avoiding direct contact and competition. This is seen as insects' preferences to certain types of basidiocarps and their asynchronous life cycles (discussed later).

\subsection{Basidiocarp life cycles}

Fungi, including polypores, have evolved strategies to withstand the change of seasons and temperatures, and temporary lack of water. We recognize four ecological groups of forest fungi (ephemeral, annual sturdy, annual hibernating and perennial) with polypores as an example group. Ephemeral polypores follow the time- and resource-saving "mushroom" strategy by developing quickly-emerging but short-lasting, small fruit bodies, which may appear only in suitable years. This safe strategy costs a limited portion of the spores, released and dispersed over a short period of time and, probably, partly eliminated before they may grow. Their basidiocarps are annual, like those of annual sturdy species which are more massive and robust, and whose both growth into full size and spore-producing period last longer. After their sporulation and death the annual sturdy fruit bodies stay on the substrate for a season or a few. Still more persistent annual hibernating species complete their growth late in the autumn, stay alive for winter season, sporulate in spring (in North Europe April-May), and then die and disintegrate. In a few cases such fruit bodies are able to continue their growth, but after the second winter they usually die out and become decomposed by insects. Species with the most robust perennial basidiocarps invest plenty of organic material in the construction of reproductive organs, but benefit from their multi-year efficiency, and their ability to sporulate repeatedly and at any time of the year.

Parmasto (1996) designated three ecological groups among polypores: annual, annual hibernating and perennial basidiomata, all different in their longevity, seasonality and the speed of decomposition. Our division into four life cycle types is essentially the same as Parmasto's, with the exception that we subdivide annual species into annual ephemeral (self-decomposing soon after brief sporulation) and annual sturdy species, whose dead fruit bodies remain on the substrate for at least the next warm season. Below we describe these ecological groups in relation to their associated beetle fauna.

This division into four types of life cycles deals with the rate of growth and the moment(s) of sporulation, and hence the span of time and changes of seasons are decisive. This division is linked with, but different from, the division into consistency classes, which illustrate the toughness, construction, moisture and physical persistence of fruit bodies. Among these factors also the hyphal system of the basidiocarp and softness/ toughness of individual hyphae appear as critical factors limiting the species composition of the associated Coleoptera. Together these all lead into major consequences in the associated beetles and in the resource partitioning of their host fungi.

The difference between the compositions of beetle species inhabiting living or dead fruit bodies was already observed by Paviour-Smith (1960). She also noted that the role of hyphal systems in both living and dead polypores may be a critical factor for fungus-dwelling generalist species of beetles. We observed the tendency of certain beetle families to be associated with unrelated polypores of a certain hyphal system. However, with the slow accumulation of the Coleoptera records from the several tens of rare and recently described polypore species, no statistically sound overall analysis can be run to test this hypothesis. We plan to discuss the microscopy of fungi and its entomological consequences in a later paper. 


\subsection{Time factor: seasonal dynamics of polypore beetles}

Records of adult insects on polypore fruit bodies are generally considered as second-class data compared to larval or rearing data. However, polypore basidiocarp as a habitat is more than just a larval food substrate. Organic sources of a forest ecosystem are consumed at various paces, from durable but nutrient-poor wood to rich but ephemeral mushrooms. The duration of larval stage and the strategy of pupation are among the most important adaptations in insects to withstand the limitations of the environment. On the extremes are on the one hand those larvae that bore wood for many years and finally pupate in the log, and on the other hand the larvae of fungus diptera with their blasting growth in mushrooms and escape to the safer soil for pupation.

Long duration is a general feature of polypore fruit bodies. The analogy with other coleopteran larval hosts may be presented so that perennial basidiocarps are decomposed in a "wood" pathway, and annual ephemeral polypores in a "mushroom" way: some beetles can pupate inside perennial or dead annual fruit bodies, like many wood-boring insects, while others require soil as a pupation medium, like the mushroom dwellers. In the race with time, basidiocarps of the annual ephemeral species avoid the majority of fungivorous Coleoptera, which are unable to complete full larval cycle during the short existence of the fruit body. Annual sturdy and hibernating species are somewhere in between the fungi with ephemeral and perennial basidiocarps. Beetle occupants of annual sturdy and hibernating polypores can utilize the same fruit bodies, during or after their sporulation, during the whole season, or up to the next spring. This substrate is dry enough to support larvae of numerous Ciidae (Sulcacis, Octotemnus, Cis comp$t u s)$, Erotylidae, and Diaperinae (Tenebrionidae). Perennial basidiocarps serve as the substrate, and become colonized and decomposed by the more slow-cycled Coleoptera, such as Dorcatominae (Anobiidae) and Bolitophagini (Tenebrionidae). These beetles are typically followed by Ciidae (Enneathron, Ropalodontus, Cis jacquemartii, C. glabratus, C. nitidus) which breed for several generations in the fruit bodies that were conditioned by their predecessors.
Consequently, one of the basic differences among the fungal hosts is the longevity and durability of both living and dead fruit bodies. These factors sort out the composition of beetle species according to their shorter or longer larval development.

The majority of polypore species live on dead wood, and only few are pathogens of living trees. Beetles developing in polypore basidiocarps duplicate this pattern: the majority of fungivorous beetle larvae utilize fruit bodies after their sporulation and death. We could show that adult Coleoptera visiting the polypores outnumber the species with fungivorous larvae (Nikitsky \& Schigel 2004). However, larvae are more effective than adult insects as consumers of the fruit body hyphae. Perennial basidiocarps of polypore species are known to grow and sporulate even for decades (Niemelä 2005), and in some cases, such as Phellinus tuberculosus, Heterobasidion parviporum, and Antrodia crassa their upper and older parts are dead and inhabited by beetles, while margin and lower side continue to grow and produce new hymenophore layers.

It is unlikely that fungi have any defence mechanisms against insects, similar to the resins of coniferous trees against scolytid beetles, and we do not know studies on fungal repellents. However, some kind of a remote olfactory evaluation of the substrate exists, which helps a beetle in search for detecting not only the fungus species, but also the growth stage of the fruitbody or its decomposition. In field experiments Octotemnus glabriculus and Cis boleti react differently to odours of young vs. mature fruit bodies of Trametes versicolor (Guevara et al. 2000). Some polypore species never host insect larvae for reasons unknown to us.

\subsubsection{Annual ephemeral fruit bodies}

The growing season of many polypores starts late, after the period when summer and autumn rains condition the wood. In Nordic countries such period may be as short as a few weeks in late August - early September, before severe frosts. The growth rate of a fruit body, even in perfect conditions, is nevertheless limited, and so the thinness of resupinate annual species makes it impossible for beetle larvae of families with larvae living inside the substrate to inhabit the basidiocarp. Anomoporia kamtschatica is an example of this 


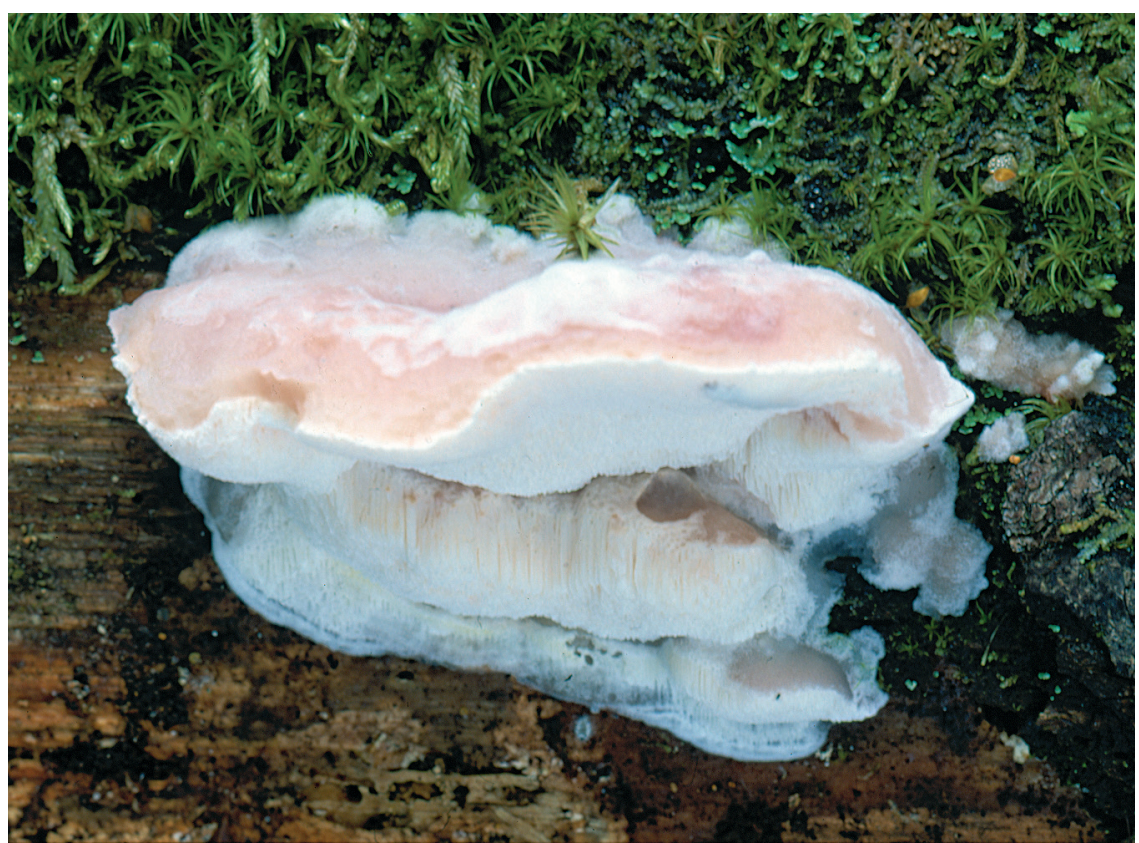

Fig. 7. Annual ephemeral basidiocarp of Leptoporus mollis. Photo TN.

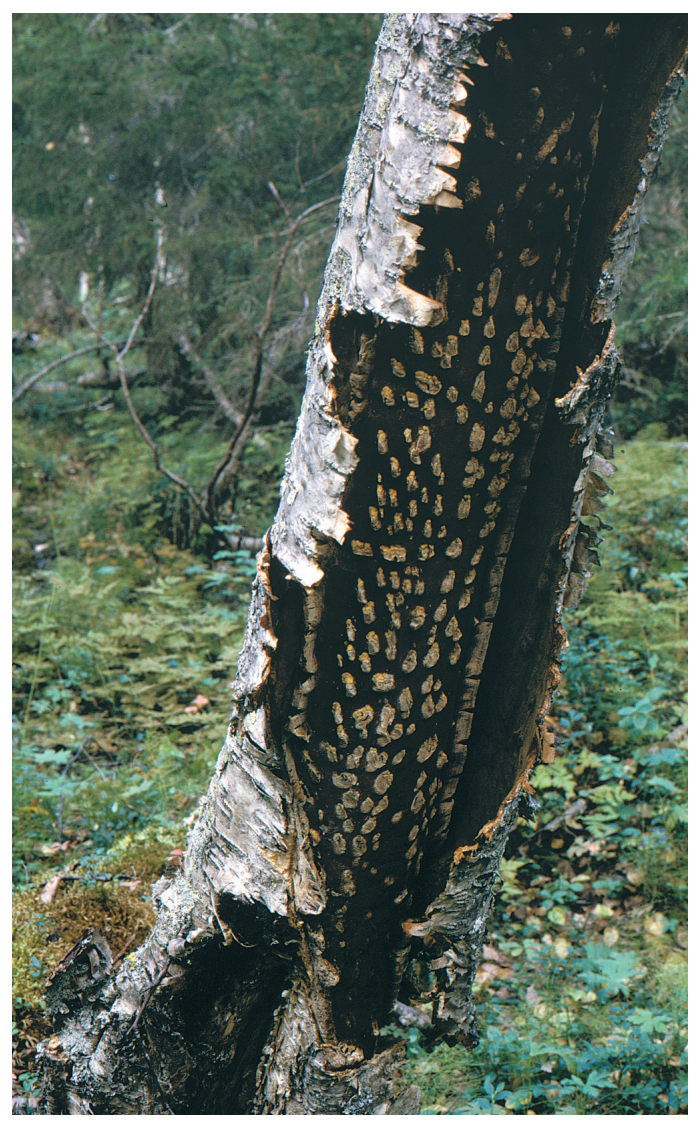

kind of fungus. Dependence on wood moisture makes the fructification of many annual polypore species irregular. During the weeks between the emergence of an annual fruit body on the substrate, and its death and decomposition during the following winter, few beetles (e.g. Hallomenus) are able to undergo full larval development. This time limit results in the dominance of adult-beetle records over larvae and rearings (Table 2, 3). Only generalist beetles with fast larval cycle are able to breed in soft and watery polypores.

Many of the annual ephemeral polypores support just scanty beetle fauna, or they are such that we do not know in Finland or elsewhere any beetle larvae to develop inside the fruit bodies. The genera Anomoporia, Ceriporia, Ceriporiopsis, Diplomitoporus, Fibroporia, Grifola, Hyphodontia, Junghuhnia, Physisporinus, Sistotrema, the majority of Skeletocutis, and Trechispora make a long, but still incomplete list of fungi with no larval records of Coleoptera. Like many other forest macrofungi, they are mostly visited by generalist adult beetles, such as Scaphisoma spp., Leptusa pulchella, Bolitochara obliqua and other Staphylinidae.

Fig. 8. Annual sturdy basidiocarp of Inonotus obliquus, growing and sporulating under detached bark of birch. Photo TN. 

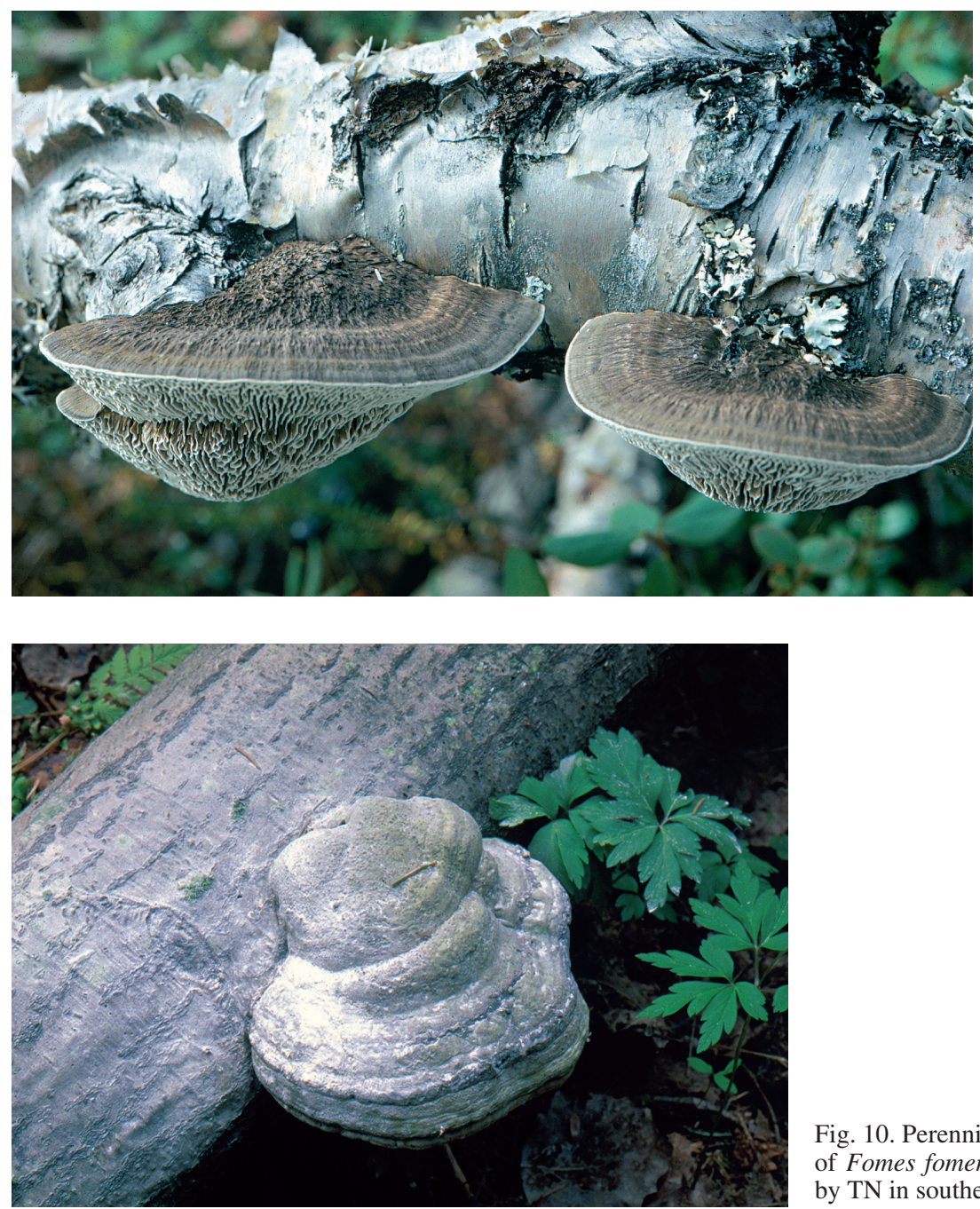

Fig. 9. Annual hibernating basidiocarp of Daedaleopsis septentrionalis. Photo TN.
Fig. 10. Perennial sporulating basidiocarp of Fomes fomentarius. Photograph taken by $\mathrm{TN}$ in southern Finland.
In western Finnish Lapland nine species (adults) visit Amylocystis lapponica, while only larvae of Hallomenus binotatus develop in it along with an unidentified Sciaridae (Diptera) species, the latter reared also from annual Junghuhnia luteoalba. Pilei of Leptoporus mollis (Fig. 7) host larvae of two Diptera species and Hallomenus axillaris, which was also reared from Postia leucomallella. Resupinate Rhodonia placenta attracts adults of five species of beetles, predominantly Phymatura brevicollis. Other fungi with few associated Coleoptera are Skeletocutis odora (4 species), Antrodia infirma (3), Rigidoporus corticola (2), Oligoporus sericeo- mollis (1), Postia lateritia (1), P. alni (1 Diptera), and Hapalopilus aurantiacus (1; Table 3).

\subsubsection{Annual sturdy fruit bodies}

Annual sturdy polypores often produce large (Laetiporus, Phaeolus, Polyporus) or numerous (Inonotus, Onnia, Climacocystis) fruit bodies. Typically these fungi sporulate the same season they grow; at the same time larvae are burrowing the basidiocarps (usually context), and adults are visiting as numerous species and individuals. The hyphal structure of the heterogeneous group of annual sturdy polypores has at least one common 
character: their pilei are rich in water when living, but after sporulation and death they become light-weight, especially if growing in sheltered places. Pilei usually keep their original shape for months even if exposed to rain and snow. Some fruit bodies (e.g. Inonotus) may contain hibernating larvae, but the majority of dwellers abandon the fungus before pupation. Few, if any beetle species come to graze or lay eggs to the overwintered basidiocarps.

Climacocystis borealis, a characteristic species of Lapland biota, attracts adult beetles of twelve species, and hosts larvae of three Coleoptera species. An excellent example of the annual sturdy polypores is Inonotus obliquus (Fig. 8). When the birch bark cracks along and the large, resupinate, subcortical fruit body starts to sporulate, a set of adult Staphylinidae become attracted, and Melandryidae larvae start to burrow in thicker patches of the fruit bodies. The used-up, rotten basidiocarp stays empty for several years, until the tall stump falls down and the secondary polypore Gloeoporus dichrous continues the decomposition of birch wood. Similar pattern was observed in Inonotus rheades (4 species of Coleoptera, larvae), Ishnoderma benzoinum (2 larvae), and Onnia leporina (2 adults). For details on the Coleoptera of Polyporus pseudobetulinus, see Schigel \& Toresson (2005).

Antrodiella pallescens (5 associated beetle species) and Gloeoporus pannocinctus (1) represent an intermediate position between annual ephemeral and annual sturdy polypores. Tough pilei of these fungi are produced in moist conditions usually close to the ground and remain for the next year partly decomposed and uninhabited.

\subsubsection{Annual hibernating fruit bodies}

Annual hibernating species, such as Bjerkandera adusta, Trametes spp., Gloeophyllum sepiarium (occasionally perennial), Polyporus brumalis, and Trichaptum abietinum are reported to sporulate during the subsequent warm season after their growth, if the winter in between is not too frosty (Parmasto 1996). Usually the fruit body of an annual hibernating species dies soon after sporulation and is colonized during the following summer. Some polypore species are traditionally regarded as annual hosts of beetle (especially Ciidae) larvae. However, their fruit bodies usually become suitable for larvae only after their sporulation and death, in about one year after they appear on the tree. Piptoporus betulinus and Daedaleopsis spp. represent such polypores with annual hibernating basidiocarps. Some species are not easily placed in either the annual sturdy or annual hibernating group, because their fruit bodies grow in a special way. In such case the new fruit body pushes out from the spot where the rotten basidiocarp of the previous year is, and as a result the living and dead basidiocarps incompletely merge together. That is typically observed in Skeletocutis odora, Trametes hirsuta, and Trichaptum spp.

Insect inhabitants of annual polypores face the necessity of yearly dispersal, because the fruit bodies are short-lasting and typically too decomposed for the next generation to utilize the parental habitat. However, annual hibernating polypores are sometimes used by Ciidae still after the second winter, especially if pilei grew in places well sheltered against rain and snow. Ephemeral nature and irregular fructification of annual polypores are the reasons for the prevalence of oligo- and polyphagous beetles over monophagous ones among the species breeding in such fungi (Nikitsky \& Schigel 2004), and for the wide range of beetle species attracted as adults. The seasonal dynamics of Trametes versicolor separates the breeding seasons of Octotemnus glabriculus (in younger fruit bodies) and Cis boleti (in fully developed basidiocarps) in Britain (Guevara et al. 2000).

Two species of the polypore genus Antrodia, A. serialis (with adults of 2 Ciidae species) and A. xantha (with Orthoperus rogeri) in western Finnish Lapland are visited when the basidiocarps are dead and partly decomposed. Dinaraea aequata and Rhizophagus dispar were found on Daedaleopsis septentrionalis (Fig. 9), a northerly polypore of mountain birch. Trametes ochracea is inhabited by a characteristic set of common beetles (Table 2).

\subsubsection{Perennial fruit bodies}

The ecological dynamics of the Coleoptera that develop in perennial fruit bodies differs from the previous types in many aspects. First, several generations of fungivorous beetles may utilize the same fruit body before dispersal becomes inevitable. Second, the basidiocarp is durable and voluminous, but hard, lasting long after its death. 
Third, the inner structure of the fruit body is complex and comprises several types of mycelial tissues (Figs. 5, 6) and thus it offers a variety of potential ecological niches for the beetle larvae. Larvae of Ciidae are generally dwelling in the context, in context-hymenophore transition, and in transitional zones between the yearly layers of hymenophore, while Dorcatoma species often avoid context and reside in the hymenophore, showing no preference to any special zones in it.

Many long-term research projects have focused on the perennial fruit bodies of common polypores and on their Coleoptera, such as Donisthorpe $(1931,1935)$, Paviour-Smith (1960), Lawrence (1973), Thunes (1994), Økland (1995), Andersen et al. (2000), Thunes et al. (2000), Siitonen et al. (2001), Ehnström \& Axelsson (2002), and Jonsell \& Nordlander (2002). Several doctoral theses, each with an extensive list of references (Nilsson 1997, Jonsell 1999, Rukke 2000, Sverdrup-Thygeson 2000) focus on the ecology of fungivory. Durable, perennial fruit bodies support a larger proportion of associated monophagous insects than what are found in more short-lasting basidiocarp types. Perennial fungi provide a continuously available resource for Coleoptera, as Jonsell \& Nordlander (2004) show with four perennial species (Fomes fomentarius, Fomitopsis pinicola, Ganoderma lipsiense, and Phellinus igniarius) vs. annual sturdy (Inonotus radiatus) and hibernating (Piptoporus betulinus) polypores. Komonen (2003) provided data on beetles of two red-listed polypores in Finland: perennial Fomitopsis rosea and annual ephemeral Amylocystis lapponica.

Some species of perennial polypores of the genera Rigidoporus and Perenniporia remain mysterious to us because just a few, if any beetles have been recorded. Coleoptera records are lacking from several Phellinus species: P. laevigatus, $P$. nigrolimitatus, $P$. tremulae, $P$. viticola, despite our sampling efforts. In addition to the collecting of beetles on rare substrates, in 2005 we started to gather data on the fauna and seasonal dynamics of beetles associated with Ganoderma lipsiense using the Kaila traps (Kaila 1993, Kaila et al. 1994) in Finland and Russia.

Our Lapland data on Heterobasidion parviporum, a perennial polypore on its northern limit of distribution, revealed one colonizing species ( $\mathrm{Cis}$ bidentatus) and two visiting ones, Gyrophaena affinis and Orthoperus rogeri. Three Ciidae spe- cies were reared from the near-threatened polypore Haploporus odorus. Adult beetles of several species were collected on the fruit bodies of Fomes fomentarius (Fig. 10), Fomitopsis rosea, and Phellinus spp. (Table 2).

\subsubsection{What happens within a polypore in the course of time?}

Any boreal forest, managed or virgin, is a habitat for many polypore species, every year producing fruit bodies of all kinds. Fungi grow, sporulate, die, become decomposed and attract beetles, and many different but simultaneous successions of Coleoptera species take place in the frugal fruit bodies. Moreover, beetle species assemblages vary from host to host, they depend on the decomposition stage of basidiocarp, and successions go at different speeds. These processes were mostly discussed in the previous sections, and are summarized in Fig. 11. Alternative host fungi are vital for insects that depend on annual and annual hibernating basidiocarps in order to support the generation continuum in bad years when the main host fungus fails to fruit.

The long-living mycelia of tyromycetoid species are producing ephemeral annual fruit bodies in late summer or early autumn, then sporulate and attract adult beetles to visit. Typically no signs of fruit bodies are seen out of the fructification season, because they die and become decomposed the same autumn they grew (Fig. 11, annual ephemeral).

Most of insect activity on the annual sturdy basidiocarps is seen during the peak of the fruit body growth and sporulation. Dead light-weight fruit bodies remain on the woody substrate for a season or two, but fewer and fewer beetles visit or colonize them in the course of time, until they disappear (Fig. 11, annual sturdy).

Annual hibernating species sporulate and die in a single, short, warm season - spring - when larvae of associated Coleoptera start eating the fruit bodies and are found together with the generation of their adult parents and visiting adults of the other beetle species. All these beetles usually decompose the whole basidiocarp by the end of the warm season. While the dead fruit bodies are consumed by beetles, new fruit bodies emerge and usually stay intact until early next year when they get consumed in their turn (Fig. 11 , annual hibernating). 
W Spring Summer Autumn Winter Spring Summer Autumn Winter Spring Summer Autumn W

Annual ephemeral: Amylocystis, Hapalopilus, Leptoporus, Postia, Pycnoporellus, Tyromyces

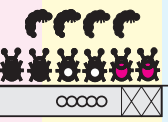

Annual sturdy: Climacocystis, Inonotus, Ishnoderma, Laetiporus, Onnia, Phaeolus, Polyporus

rerer

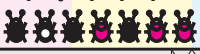

Annual hibernating: Bjerkandera, Daedaleopsis, Funalia, Piptoporus, Trametes, Trichaptum

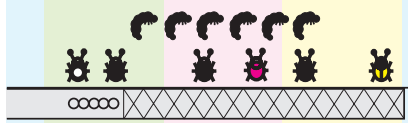

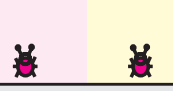
rerer

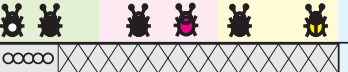

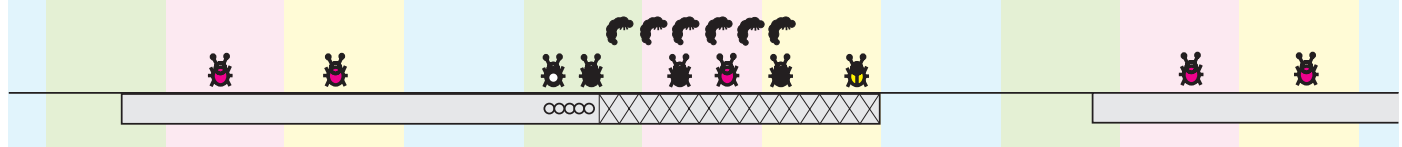

Perennial: Fomes, Fomitopsis, Ganoderma, Heterobasidion, Phellinus

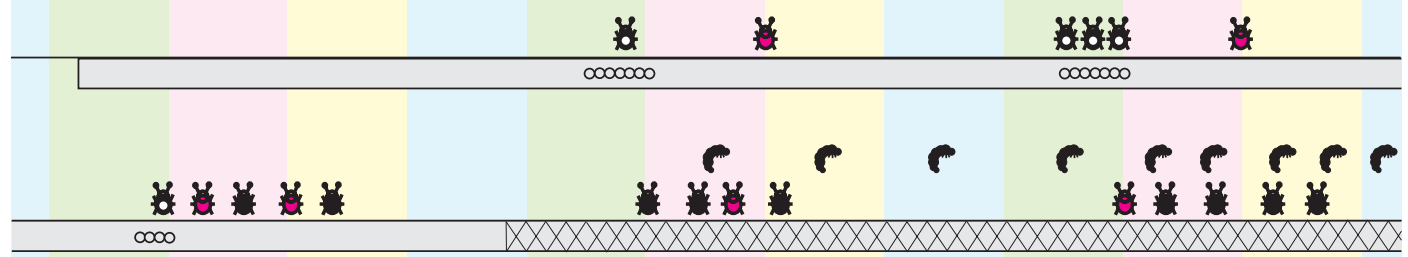

rerrererrerrererererer

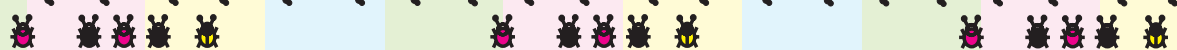

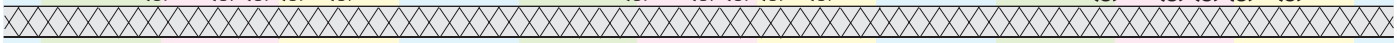


Similarly the perennial basidiocarps are growing at first more or less intact, and start to attract adult beetles mostly during the sporulation. It may take several years and even decades before the first larva appears to burrow its tunnel. Like annual hibernating species, also the perennial ones typically face a larval attack after their death, but it takes still several years until the fruit body is completely decomposed (Fig. 11, perennial). An additional set of insect species utilize anamorphic fungi that grow over the dead basidiocarps.

\subsection{Sporulation of polypores, and spore-attracted beetles}

In the yearly cycle of polypore's fruit-body growth, the species composition of visiting adult beetles changes drastically at the sporulation time. This period differs in its duration and seasonal schedule according to the polypore species, and fluctuates from year to year. The period of active spore productivity of a polypore fungus is usually short, a few weeks (Fig. 11). The instability of sporulation was observed also in constant weather conditions, even though the spore release is ruled by air temperature and humidity. One square centimetre of pore surface may shed $10^{8}$ spores per day, and seasonal outcome can be as much as $5 \times 10^{11}$ spores (Parmasto \& Parmasto 2003). Adult beetles utilize the energyrich spores that deposit below and above the basidiocarp.

Spore-feeding beetles are mostly recorded in spring and early summer. Larger perennial species often produce bright and voluminous spore masses: the light-grey powder of spores, covering the trunk and ground below the pileus of Fomes fomentarius can be seen from a distance (Fig. 10), as well as the cocoa-brown spore dust of Ganoderma lipsiense. Adult Latridiidae, Corylophidae, and Staphylinidae are among the commonest spore-eaters. Epuraea species visit the annual hibernating Daedaleopsis spp. during their sporulation in early spring.

Unlike the perennial species with easily recognised sporulation periods, annual ephemeral species have smaller and often hidden fruit bodies, and their sporulation time is not easily observed in the field. Few, if any, beetles are collected during this time. The terms of our polypore inventories took place in early autumn in order to record such polypore species and gain data on associated Coleoptera. As a result, beetle records in Table 2 are more detailed in regard of annual species of polypores, such as Amylocystis lapponica, Climacocystis borealis, and Rhodonia placenta. For the same reason, almost a half of the Coleoptera (Table 3) are Staphylinidae. Several species of this family showed a tendency of using spores and decomposing basidiocarps, at least as a part of their imago diet, but more observations are needed to verify this feeding habit.

\subsection{Feeding strategies of fungivorous Coleoptera}

Beetles which are found on polypores can be placed in one of the two major groups: either those with fungivorous larvae, i.e. dwellers, or those with non-fungivorous larvae, i.e. visitors (Table 5). By the term dweller we mean bee-

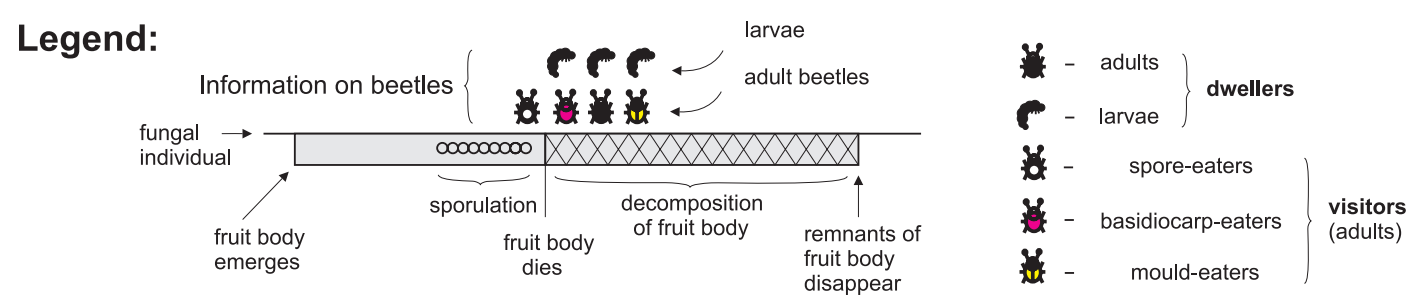

Fig. 11 (this and previous page). Annual ephemeral, annual sturdy, annual hibernating and perennial fruit bodies of polypores, their seasonality and attracted beetles: a simplified calendar. The consequent years are divided in four seasons, respectively coloured. Simultaneous presence of the living and dead fruit bodies of the same polypore individual are not shown for clarity. 
tles which spend most of their life cycle inside the fruit-body and leave the fungus usually for mating and dispersal only. Alternatively, a visitor uses fruit body host as an adult beetle, but its larvae live elsewhere. Visitors may be fungivorous, or not. Fungivorous visitors can be still subdivided into three groups according to their food type: spore-eaters, basidiocarp-eaters, and mould-eaters. All these groups are independently characterized by their feeding strategies (Lawrence 1989) as macrophagous, i.e. feeding on solid mycelial tissues (dwellers, basidiocarp-eaters) $v s$. microphagous, which feed on spores and loose hyphae (spore- and mould-eaters). Dwellers and visitors differ in their order of appearance, their importance in decomposition process, their ability to utilize fungal substrate, and their species diversity (Table 5).

The process of basidiocarp colonization can be simplified as follows: the first to appear on the fungal substrate are spore-eaters (few species, very weak destructors), followed by basidiocarpeaters (plenty of species, weak destructors), and dwellers (few species, effective destructors). Finally, at the late stages of decomposition, anamorphic fungi grow over the rotting fruit body, and they host another wave of microphagous Coleoptera, such as Corticaria lapponica on Fomes fomentarius, or Orthoperus rogeri, Orthoperus corticalis, and Corticarina lambiana on Polyporus pseudobetulinus (Table 3 ). In nature the successions are influenced by environmental factors. Species compositions of insect assemblages and their temporal and functional structures vary (Fig. 11). Modifications of the described pattern are countless, and most probably no basidiocarp is decomposed exactly like the other one.

Species composition and ecology of Coleoptera attracted by spores and anamorphic fungi remain still poorly known, and require further studies. Spores and secondary anamorphic fungi are easily overlooked and short-persisting, but anyhow characteristic habitats consorted with specialized Coleoptera.

Forest ecosystem is a carbohydrate horn-ofplenty, but protein desert, and fungi are among the few compact, common and voluminous food sources (Fig. 12). Even among the Dermetistidae with their specialisation to dry animal remnants, species of Orphilus are found to feed on brown rot caused by fungi (Zhantiev 2001), though not on the polypore fruit bodies themselves. There is little doubt that fungivory in the order Coleoptera evolved independently several times. The present-time set of beetle genera attracted to polypores, and feeding strategies of their nonfungivorous relatives within families point towards such multiple development.

Adaptations in larvae (the main feeding lifestage of Coleoptera) give the most reliable clues for explaining the origins of mycophagy among beetles. Practically every family of polyporefeeding beetles also contains taxa with non-fungal (non-basidiocarp) diet. Most of these families contain genera of beetles both with burrowing fungivorous larvae, and genera with different, more family-characteristic feeding strategies. This is the case in Anobiidae (Dorcatominae in polypores, others mostly in wood), and Melandryidae (fungivorous Hallomenus, Abdera, Orchesia and Zilora). Saprotrophic larvae of Tenebrionidae typically feed on plant remnants, but fungivorous Diaperini and Bolitophagini colonize dead basidiocarps. Their fungivory probably developed from wood-eating ancestors: the wood-to-polypore evolution.

Some families, like Ciidae and Erotylidae comprise mainly fungivorous forms and the evolution of their fungivory is difficult to see. Mushrooms host insects with fast-developing larvae, e.g. mobile surface-living larvae of Staphylinidae, or burrowing larvae of Diptera. Species diversity and abundance of fungivorous Staphylinidae and Diptera reach their peak in agarics and boletes, but some of these insects live on polypores: the mushroom-to-polypore evolution.

Adult beetles usually have more variable diets than larvae. Dwellers (Table 5) are often found feeding on the substrate they use for copulation and oviposition. However, adult microphagous beetles (both spore- and mould-eaters) feed on polypores, but their larvae are typically found elsewhere: larvae utilize a broad variety of food substrates, including sap and rotten fruits (Nitidulidae), carrion (Cholevidae), myxomycetes (Leiodidae, Sphindidae, Latriidae), and anamorphic fungi (Latridiidae). Adult Trogossitidae are often collected on the hymenophore of various polypore species, but Thymalus has fungivorous larvae, and those of Peltis and Ostoma live in brown rot. Indirect co-evolution between fungi and associated predators, omnivorous insects, and parasitoids requires more data on the ecology of individual species. Nordic Saproxylic 
Table 5. Feeding strategies of fungivorous Coleoptera and their role in the decomposition of polypore fruit bodies. Terms are described in the text (section Feeding strategies of fungivorous Coleoptera). Column titles are abbreviated as follows: Feeding strategy $=$ feeding strategy of fungivorous Coleoptera, Life stage = dominant life stage of Coleoptera, Food char. = food characteristics, macrophagy (solid mycelium tissue) vs. microphagy (spores, loose hyphae), Decomp. stage $=$ corresponding decomposition stage of basidiocarp (Schigel et al. 2004), Influence = beetles' ability to decompose the basidiocarp, No. of species = estimate of relative beetle species diversity.

\begin{tabular}{|c|c|c|c|c|c|c|}
\hline $\begin{array}{l}\text { Feeding } \\
\text { strategy }\end{array}$ & $\begin{array}{l}\text { Life } \\
\text { stage }\end{array}$ & $\begin{array}{l}\text { Food } \\
\text { char. }\end{array}$ & $\begin{array}{l}\text { Decomp. } \\
\text { stage }\end{array}$ & Influence & $\begin{array}{l}\text { No. of } \\
\text { species }\end{array}$ & Examples \\
\hline Dwellers & $\begin{array}{l}\text { Larvae \& } \\
\text { adults }\end{array}$ & Macro & $3-4$ & Strong & Medium & $\begin{array}{l}\text { Ciidae, Anobiidae (Dorcatominae), Ten- } \\
\text { ebrionidae (Diaperini, Pentaphyllini and } \\
\text { Bolitophagini), Erotylidae, Melandryi- } \\
\text { dae (Hallomenus, Orchesia, Abdera, and } \\
\text { Zilora), Tetratomidae, Mycetophagidae, } \\
\text { Trogossitidae (Thymalus) }\end{array}$ \\
\hline
\end{tabular}

\section{Visitors}

\begin{tabular}{|c|c|c|c|c|c|c|}
\hline $\begin{array}{l}\text { Spore- } \\
\text { eaters }\end{array}$ & Adults & Micro & $1(2)$ & Almost no & Low & $\begin{array}{l}\text { Nitidulidae (Epuraea and Glishro- } \\
\text { chilus), Latridiidae, Leiodidae (Anisoto- } \\
\text { ma and Agathidium) }\end{array}$ \\
\hline $\begin{array}{l}\text { Basidiocarp } \\
\text { eaters }\end{array}$ & Adults & Macro & $2(1)$ & Weak & High & $\begin{array}{l}\text { Staphylinidae, Trogossitidae (Ostoma } \\
\text { and Peltis) }\end{array}$ \\
\hline $\begin{array}{l}\text { Mould- } \\
\text { eaters }\end{array}$ & Adults & Micro & 4 & Almost no & Low & $\begin{array}{l}\text { Corylophidae, Latridiidae, Cryptopha- } \\
\text { gidae, Sphindidae }\end{array}$ \\
\hline
\end{tabular}

Network (Stokland et al. 2006, Schigel 2005) is currently working towards a major database on saproxylic insects in Fennoscandia. New phylogenies developed in both fungi and insects may well lead into new views on their co-evolution.

\subsection{Notes on selected insects}

In our study in western Finnish Lapland, beetle larvae of just a few species were found to utilize basidiocarps of many polypore species: $\mathrm{Cis}$ bidentatus (reared from 7 species of polypores), Dorcatoma dresdensis (3) and Cis jacquemartii (3). These numbers are influenced by the limitations of our collecting method: the commonest polypore species were not sampled, and e.g. Fomes fomentarius is not listed for Cis jacquemartii in Table 3. For references on beetles in common polypores, see section Perennial fruit bodies (above). Cis comptus was found to breed in the pilei of Haploporus odorus, an exceptional perennial species among the beetle's preferred annual substrates. This fragrant white polypore of goat willow also hosts larvae of Dolichocis laricinus and Cis bidentatus. Larvae of some Melandryidae (Hallomenus) are confined to tyromycetoid polypores, such as Leptoporus mollis, Amylocystis lapponica, and Postia spp., while others (Abdera, Orchesia) utilize the living or recently died basidiocarps of annual sturdy Inonotus and are hardly ever found in decomposed and overwintered fruit bodies.

Leptoporus mollis, a polypore with tyromycetoid consistency, hosts larvae of Bolitophila occlusa (reared also from soft Postia alni) and one unidentified Sciridae species (reared also from Amylocystis lapponica). Sciophila buxtoni is a diptera species linked with the more tough but annual species of polypores, Antrodiella pallescens and Junghuhnia luteoalba.

A number of Staphylinidae species that visit the fruit bodies of polypores were found in all three protected forests: Acrulia inflata, Atheta aenipennis, A. crassicornis, A. fungi, A. picipes, Deliphrum tectum, Leptusa pulchella, Lordithon lunulatus, L. speciosus, L. thoracicus, Omalium rivulare, O. strigicolle, Phyllodrepa linearis, Scaphisoma subalpinum, Tachinus laticollis. The majority of these species are common all over Finland, as seen from species' frequency scores of 15 or less (Table 3). Some species of the cluster are generalists as adults and show a wide range of hosts: Acrulia inflata (7 species of polypores), Atheta picipes (4), Lordithon lunulatus (3), L. speciosus (3). All the rest of the Staphylinidae were collected from just one or two polypore species. Among the indiscriminate non-staphylinid beetles we acknowledge $\mathrm{Or}$ - 


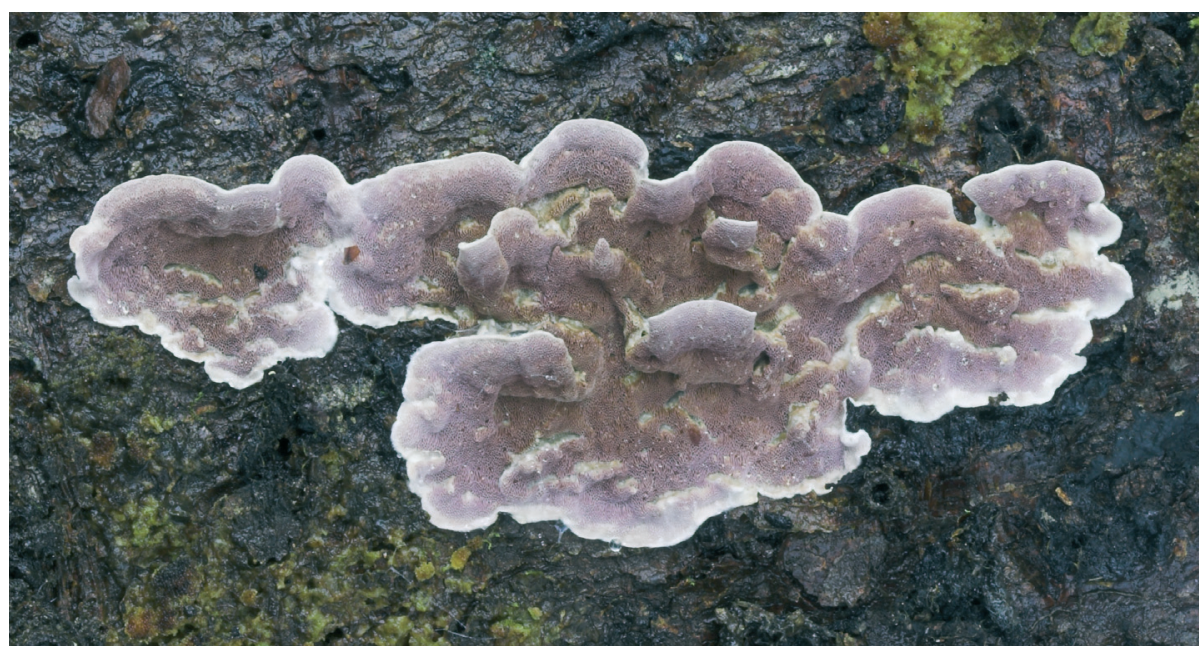

Fig. 12. Skeletocutis lilacina, a vulnerable polypore of northern taiga. Photo DSS.

thoperus rogeri (6 polypore species, mostly decomposed fruit bodies), Rhizophagus dispar (5), Cis dentatus (5), Dolichocis laricinus (4). The less frequent species (score 30 or higher) have narrow range of hosts: Phymatura brevicollis (2 species of polypores), Oxypoda hansseni (1), Lordithon trimaculatus (1), A. boletophila (1). A single imago of Pytho kolwensis (EN) was noted at the forest margin during the Pisavaara inventory in 2003 .

\section{Conclusion}

Data on fungivorous beetles collected in the field, or obtained by rearing, tends to be biased towards the commonest generalists and the most prolific specialists. Between these extremes, there are fungus-attracted species that are overlooked and undercollected. The chance to collect a generalist beetle is high because it is found on many fungi which collectively are numerous enough to be frequently spotted. Specialists are found on common and continuously available fungi, and are often collected, too: Cis glabratus and Dorcatoma punctulata in Fomitopsis pinicola, Cis jacquemartii in Fomes fomentarius, Cis nitidus in Ganoderma lipsiense, and Eledona agricola in Laetiporus sulphureus. The attraction by polypores of adult beetles is an undeservedly ignored component of species ecology, and requires further studies in order to precisely document the interspecific relations within one of the most diverse groups of organisms in Nordic forests: the saproxylic species.

Acknowledgements: We are grateful for the identification of parts of Coleoptera material to Nikolai B. Nikitsky (Moscow State University), Aleksander V. Kompantsev (A.N. Severtsov Institute of Ecology and Evolution, Moscow), and Viktor B. Semenov (E. I. Marcinovsky Institute of Medical Parasitology and Tropical Medicine, Moscow). Diptera were examined by Jevgeniy B. Jakovlev (Finnish Forest Research Institute), for which we thank. Heikki Kotiranta (Finnish Environment Institute) kindly identified some of corticioid fungi. The Finnish Forest and Park Service warmly hosted us during species inventories. We are grateful to Päivi Paalamo, Heikki Eeronheimo, and Yrjö Norokorpi (Lapin luontopalvelut) for organizing the polypore inventories. Mats Jonsell (Swedish University of Agricultural Sciences) kindly shared his experience and library with us. Juha Siitonen (Finnish Forest Research Institute) and members of the Finnish Coleoptera mailing list "Kuoriaisposti" made valuable comments. The study was generously supported by longterm grants from the Ministry of Environment, Finland (PUTTE: the research programme for deficiently known and threatened forest species). 


\section{References}

Ahti, T., Hämet-Ahti, L. \& Jalas, J. 1968: Vegetation zones and their sections in northwestern Europe. - Ann. Bot. Fennici 5: 169-211.

Andersen, J., Olberg, S. \& Haugen, L. 2000: Saproxylic beetles (Coleoptera) of Troms and western Finnmark, northern Norway, with exceptional distribution in Fennoscandia. - Norwegian J. Entomol. 47: 29-40.

Berglund, H. \& Ryvarden, L. 2000: Oligoporus norrlandicus nov. sp. - Cryptogamie Mycol. 21: 145-146.

Donisthorpe, H. 1931: Coleoptera found in the "birch bracket" fungus Polyporus betulinus. - Entomol. Res. 43: 14 .

Donisthorpe, H. 1935: The British fungicolous Coleoptera. - Entomol. Monthly Mag. 71: 21-31.

Ehnström, B. \& Axelsson, R. 2002: Insektsgnag i bark och ved. - ArtDatabanken SLU: 165-498.

Eriksson, J. \& Strid, A. 1969: Studies in Aphyllophorales (Basidiomycetes) of Northern Finland. - Ann. Univ. Turku A2 40 (Rep. Kevo Subarct. Sta. 4): 112-158.

Guevara, R., Hutcheson, K.A., Mee, A.C., Rayner, A.D.M. \& Reynolds, S.E. 2000: Resource partitioning of the host fungus Coriolus versicolor by two ciid beetles: the role of odour compounds and host ageing. - Oikos 91: 184-194.

Jonsell, M. 1999: Insects in wood-decaying polypores: conservation aspects. $\mathrm{PhD}$ thesis. - Acta Univ. Agric. Sueciae Silvestria 93: 1-47.

Jonsell, M. \& Nordlander, G. 2002: Insects in polypore fungi as indicator species: a comparison between forest sites differing in amounts and continuity of dead wood. - Forest Ecol. Managem. 157: 101-118.

Jonsell, M. \& Nordlander, G. 2004: Host selection patterns in insects breeding in bracket fungi. - Ecol. Entomol. 29: 697-705.

Kaila, L. 1993: A new method for collecting quantitative samples of insects associated with wood fungi. - Entomol. Fennica 4: 21-23.

Kaila, L., Martikainen, P., Punttila, P. \& Yakovlev, E.B. 1994: Saproxylic beetles (Coleoptera) on dead birch trunks decayed by different polypore species. - Ann. Zool. Fennici 31: 97-110.

Kinnunen, J. \& Niemelä, T. 2005: North European species of Ceriporiopsis (Basidiomycota) and their Asian relatives. - Karstenia 45: 81-90.

Komonen, A. 2003: Insects in wood-decaying fungi: ecology, diversity and response to forest management. $\mathrm{PhD}$ thesis. - Univ. Joensuu. $35 \mathrm{pp}$.

Lawrence, J.F. 1973: Host preference in Ciid beetles (Coleopetra: Ciidae) inhabiting the fruiting bodies of Basidiomycetes in North America. - Bull. Mus. Comparative Zool. 145: 163-212.

Lawrence, J.F. 1989: Mycophagy in the Coleoptera: Feeding strategies and morphological adaptations. - In: Wilding, N., Collins, N.M., Hammond, P.M. \& Webber, J.F. (eds.), Insect-fungus interactions. 14 Symposium of the Royal Entomological Society of London in collaboration with the British Mycological Society, 16-17 September 1987, London. Academic Press, London: 1-23.
Lindberg, H. \& Saris, N.E. 1952: Insektsfaunan i Pisavaara naturpark (Finland, Prov. Ob.). - Acta Soc. Fauna Flora Fennica 69: 1-82.

Matthewman, W.G. \& Pielou, D.P. 1971: Arthropods inhabiting the sporophores of Fomes fomentarius (Polyporaceae) in Gatineau Park, Quebec. - Canadian Entomologist 103: 775-847.

Miettinen, O., Niemelä, T. \& Spirin, W. 2006: Northern Antrodiella species, the identity of A. semisupina, and type studies of related taxa. - Mycotaxon 96: 211-239.

Müller, C., Jaeger, B., Kompantsev, A.V. \& Uhlig, M. 2001: Type and species catalogue of the minute tree-fungus beetles of the Museum für Naturkunde in Berlin, with general information on the Coleoptera collection, its curation and "Historical collection" (Coleoptera, Polyphaga, Ciidae and Pterogeniidae). - Mitt. Mus. Naturkund. Berlin, Zool. Reihe 77: 303323.

Niemelä, T. 1998: The Skeletocutis subincarnata complex (Basidiomycetes), a revision. - Acta Bot. Fennica 161: 1-35.

Niemelä, T. 2003: What is the commonest polypore in Finland? - Sienilehti 55: 46-53.

Niemelä, T. 2005: Käävät, puiden sienet. Polypores, lignicolous fungi. - Norrlinia 13: 1-320.

Niemelä, T. \& Dai, Y.C. 1998: Luoston suojelualueen kääpäinventointi 1998. - Metsähallitus, Perä-Pohjolan luontopalvelut, Helsinki. 32 pp.

Niemelä, T. \& Dai, Y.C. 1999: Polypores of Luosto. - Nature Protection Publ. Finnish Forest Park Service, Ser. A 105: 1-59.

Niemelä, T., Dai, Y.C., Kinnunen, J. \& Schigel, D.S. 2004: New and in North Europe rare polypore species (Basidiomycota) with annual, monomitic basidiocarps. - Karstenia 44: 67-77.

Niemelä, T. \& Kinnunen, J. 2001: Korouoman suojelualueen kääpäinventointi 2001. - Metsähallitus, PeräPohjolan luontopalvelut, Helsinki. 38 pp.

Niemelä, T. \& Kinnunen, J. 2002: Ylläksen-Aakenuksen suojelualueen kääpäinventointi 2001. - Metsähallitus, Perä-Pohjolan luontopalvelut, Helsinki. 30 pp.

Niemelä, T. \& Kinnunen, J. 2003: Pisavaaran luonnonpuiston kääpäinventointi 2003. - Metsähallitus, PeräPohjolan luontopalvelut, Helsinki. 16 pp.

Niemelä, T., Kinnunen, J. \& Dai Y.C. 2003a: Käävät. In: Ylläs-Aakenuksen alueen luonto. - Metsähallituksen luonnonsuojelujulkaisuja A 141: 84-107, 183-186.

Niemelä, T., Kinnunen, J. \& Kotiranta, H. 2005a: Pisavaaran luonnonpuiston ja Korouoman - Jäniskairan suojelualueen käävät. - Metsähallituksen luonnonsuojelujulkaisuja, Ser. A 150: 1-51.

Niemelä, T., Kinnunen, J., Larsson, K.H., Schigel, D.S. \& Larsson, E. 2005b: Genus revisions and new combinations of some North European polypores. - Karstenia 45: 75-80.

Niemelä, T., Kinnunen, J., Lindgren, M., Manninen, O., Miettinen, O., Penttilä, R. \& Turunen, O. 2001: Novelties and records of poroid Basidiomycetes in Finland and adjacent Russia. - Karstenia 41: 1-21.

Niemelä, T., Kinnunen, J. \& Manninen, O. 2002: Koitajoen Natura-alueen kääpäinventointi 2002. - Metsähallitus, Itä-Suomen luontopalvelut, Helsinki. 41 pp. 
Niemelä, T., Kinnunen, J., Renvall, P. \& Schigel, D. 2003b: Phellodon secretus (Basidiomycota), a new hydnaceous fungus from northern pine woodland. - Karstenia 43: 37-44.

Niemelä, T., Wallenius, T. \& Kotiranta, H. 2002: The kelo tree, a vanishing substrate of specified wood-inhabiting fungi. - Polish Bot. J. 47: 91-101.

Nikitsky, N.B. \& Schigel, D.S. 2004: Beetles in polypores of the Moscow region: checklist and ecological notes. - Entomol. Fennica 15: 6-22.

Nilsson, T. 1997: Spatial population dynamics of the black tinder fungus beetle Bolitophagus reticulatus (Coleoptera: Tenebrionidae). - Acta Univ. Upsaliensis (Comprehensive Summaries of Uppsala Dissertations from the Faculty of Science and Technology) 311: $1-44$.

Økland, B. 1995: Insect fauna compared between six polypore species in a southern Norwegian spruce forest. - Fauna Norvegica B 42: 21-46.

Parmasto, E. 1970: The Lachnocladiaceae of the Soviet Union. - Inst. Zool. Bot., Tartu. 163 pp.

Parmasto, E. 1973: Novyi rod Inonotopsis Parm., sem. Hymenochaetales. - Folia Cryptog. Estonica 2:1113.

Parmasto, E. 1996: Hymenomycetes with hibernating basidiomata. - Fungi and lichens in the Baltic region. Abstracts of the 13th International Conference on Mycology and Lichenology, Tartu: 41-42.

Parmasto, E. 2004: Distribution maps of Estonian fungi 3, pore fungi. - Scripta Mycologica 19:1-224.

Parmasto, E. \& Parmasto, I. 2003: Spore productivity of polypores. - Abstracts of the XIV Congress of European Mycologists, Yalta: 51-52.

Paviour-Smith, K. 1960: The fruiting bodies of macrofungi as habitats for beetles of the family Ciidae (Coleoptera). - Oikos 11: 43-71.

Rassi, P. (ed.) 1993: Suomen kovakuoriaisten (Coleoptera) frekvenssipisteet 1.1.1960 - 1.1.1990. - Maailman Luonnon Säätiön WWF Suomen Rahaston Raportteja 6: 1-136.

Rassi, P., Alanen, A., Kanerva, T. \& Mannerkoski, I. (eds.) 2001: The 2000 Red List of Finnish species. Ministry of the Environment \& Finnish Environment Institute: $1-432$.

Rayner, A.D.M. \& Boddy, L. 1988: Fungal decomposition of wood. - John Wiley, Chichester. 603 pp.

Renvall, P. 2005: Taxonomy of the Oligoporus hibernicus complex (Basidiomycota), with the new species Oligoporus parvus. - Karstenia 45: 91-102.

Rintala, T., Ahlroth, P., Hyvärinen, E., Mattila, J., Nevalainen, J., Siitonen, J. \& Toivanen, T. 2003: Hyönteiset. In: Ylläs-Aakenuksen alueen luonto. - Metsähallituksen luonnonsuojelujulkaisuja A 141: 153-161, 203-213.

Rukke, B.A. 2000: The significance of habitat fragmentation, spatial scale and microhabitat variables for dead wood fungi beetles. - Ser. Dissert. Fac. Mat. Nat. Sci., Univ. Oslo 58: 1-17.
Ryvarden, L. \& Gilbertson, R.L. 1993: European polypores 1: Abortiporus-Lindtneria. - Synopsis Fungorum 6: 1-387.

Schigel, D.S. 2005: Links of the saproxylic chain in European boreal forests. - Fungi in natural and anthropogenic ecosystems: Proceedings of the International Conference dedicated to the centenary of the beginning by professor A.S. Bondarcev his research activity at the V.L. Komarov Botanical Institute RAS (24-28 April, 2005, Saint Petersburg) 2: 335-337.

Schigel, D.S., Niemelä, T., Similä, M., Kinnunen, J. \& Manninen, O. 2004: Polypores and associated beetles of the North Karelian Biosphere Reserve, eastern Finland. - Karstenia 44: 35-56.

Schigel, D.S. \& Toresson, H.G. 2005: New records of Polyporus pseudobetulinus, a rare polypore fungus (Basidiomycota, Aphyllophorales) in Scandinavia, and notes on associated beetles. - Memoranda Soc. Fauna Flora Fennica 81: 102-107.

Siitonen, J., Penttilä, R. \& Kotiranta, H. 2001: Coarse woody debris, polyporous fungi and saproxylic insects in an old-growth forest in Voldozero National Park, Russian Karelia. - Ecol. Bull. 49: 231-242.

Silfverberg, H. 2004: Enumeratio nova Coleopterorum Fennoscandiae, Daniae et Baltiae. - Sahlbergia 9: $1-111$.

Sippola, A.L., Siitonen, J. \& Kallio, R. 1995: Faunistics of Coleoptera in subarctic pine forests in Finnish Lapland. - Entomol. Fennica 6: 201-210.

Sörensson, M. 1997: Morphological and taxonomical novelties in the world's smallest beetles, and the first Old World record of Nanosellini (Coleoptera: Ptiliidae). - Systematic Entomol. 22: 257-283.

Stokland, J.N., Dahlberg, A., Meyke, E. Schigel, D.S. \& Siitonen, J.: The Nordic saproxylic database - a comprehensive overview of the biological diversity in dead wood. - I European Congress of Conservation Biology, Hungary, 22-26 August 2006: 159.

Sverdrup-Thygeson, A. 2000: Forest management and conservation: woodland key habitats, indicator species and tree retention. - Ser. Dissert. Fac. Mat. Nat. Sci., Univ. Oslo 56: 1-32.

Thunes, K.H. 1994: The coleopteran fauna of Piptoporus betulinus and Fomes fomentarius (Aphyllophorales: Polyporaceae) in western Norway. - Entomol. Fennica 5: 157-168.

Thunes, K.H., Midtgaard, F. \& Gjerde, I. 2000: Diversity of coleoptera of the bracket fungus Fomitopsis pinicola in a Norwegian spruce forest. - Biodiversity Conservation 9: 833-852.

Wegelius, A. 1960: Bidrag till kännedomen om skalbaggsfaunan inom Pallas-Ounastunturi nationalpark. - Notulae Entomol. 40: 86-107.

Zhantiev, R.D. 2001: Dermestids of the genus Orphilus Er. (Coleoptera, Dermestidae) of Palaearctic fauna. - Entomological Review 80: 611-619. 